\title{
Serum and urine metabolomics reveal potential biomarkers of T2DM patients with nephropathy
}

\author{
Mingmei Shao ${ }^{1,2 \#}$, Hao Lu ${ }^{3 \#}$, Ming Yang ${ }^{4}$, Yang Liu ${ }^{2}$, Peihao Yin ${ }^{5}$, Guowen $\mathrm{Li}^{6}$, Yunman Wang ${ }^{5}$, Lin Chen ${ }^{5}$, \\ Qingguang Chen ${ }^{3}$, Cheng Zhao ${ }^{6}$, Qun $\mathrm{Lu}^{6}$, Tao Wu ${ }^{1,2}$, Guang Ji ${ }^{2}$ \\ ${ }^{1}$ Institute of Interdisciplinary Integrative Medicine Research, Shanghai University of Traditional Chinese Medicine, Shanghai 201203, China; \\ ${ }^{2}$ Institute of Digestive Disease, Longhua Hospital, Shanghai University of Traditional Chinese Medicine, Shanghai 200032, China; ${ }^{3}$ Department of \\ Endocrinology and Metabolism, Shuguang Hospital Affiliated to Shanghai University of Traditional Chinese Medicine, Shanghai 201203, China; \\ ${ }^{4}$ Department of Good Clinical Practice Office, Longhua Hospital, Shanghai University of Traditional Chinese Medicine, Shanghai 200032, China; \\ ${ }^{5}$ Putuo Hospital, Shanghai University of Traditional Chinese Medicine, Shanghai 200062, China; ${ }^{6} \mathrm{Pharmacy}$ Department, Shanghai TCM-integrated \\ Hospital, Shanghai University of Traditional Chinese Medicine, Shanghai 200071, China \\ Contributions: (I) Conception and design: T Wu, G Ji; (II) Administrative support: T Wu, G Ji; (III) Provision of study materials or patients: M \\ Shao, H Lu; (IV) Collection and assembly of data: M Shao, H Lu, P Yin, G Li, Y Wang, L Chen, Q Chen, C Zhao, Q Lu; (V) Data analysis and \\ interpretation: M Shao, H Lu, M Yang, Y Liu; (VI) Manuscript writing: All authors; (VII) Final approval of manuscript: All authors. \\ \#These authors contributed equally to this work. \\ Correspondence to: Prof. Guang Ji, PhD. Institute of Digestive Disease, Longhua Hospital, Shanghai University of Traditional Chinese Medicine, South \\ Wanping Road 725, Shanghai 200032, China. Email: jiliver@vip.sina.com; Dr. Tao Wu, PhD. Institute of Interdisciplinary Integrative Medicine \\ Research, Shanghai University of Traditional Chinese Medicine, Cailun Road 1200, Shanghai 201203, China. Email: wutao001827@163.com.
}

Background: Diabetes is a metabolic disease and is often accompanied by severe microvascular and macrovascular complications. A comprehensive understanding of its complex mechanisms can help prevent type 2 diabetes mellitus (T2DM) complications, such as diabetic nephropathy (DN).

Methods: To reveal the systemic metabolic changes related to renal injury, clinical information of T2DM patients with or without nephropathy was collected, and it was found that serum urea levels of DN patients were significantly higher in T2DM patients without nephropathy. Further along the disease progression, the serum urea levels also gradually increased. We used gas chromatograph coupled with time-of-flight mass spectrometry (GC-TOFMS) metabolomics to analyze the serum and urine metabolites of T2DM patients with or without nephropathy to study the metabolic changes associated with the disease.

Results: Finally, we identified 61 serum metabolites and 46 urine metabolites as potential biomarkers related to $\mathrm{DN}(\mathrm{P}<0.05$, VIP $>1)$. In order to determine which metabolic pathways were major altered in $\mathrm{DN}$, we summarized pathway analysis based on $\mathrm{P}$ values from their impact values and enrichment. There were 9 serum metabolic pathways and 12 urine metabolic pathways with significant differences in serum and urine metabolism, respectively.

Conclusions: This study emphasizes that GC-TOFMS-based metabolomics provides insight into the potential pathways in the pathogenesis and progression of DN.

Keywords: Type 2 diabetes mellitus (T2DM); diabetic nephropathy; gas chromatograph coupled with time-offlight mass spectrometry (GC-TOFMS); metabolomics

Submitted Oct 19, 2019. Accepted for publication Jan 02, 2020.

doi: $10.21037 /$ atm.2020.01.42

View this article at: http://dx.doi.org/10.21037/atm.2020.01.42 


\section{Introduction}

According to the International Diabetes Federation in 2017, 114 million (10.9\%) Chinese have been diagnosed with diabetes in the last 20 years, and by 2045, the number is expected to be 119 million (11.6\%) (1). The incidence and prevalence of diabetes have increased markedly, especially for type 2 diabetes mellitus (T2DM). A vast number of people with T2DM have progressed toward different complications in recent decades. Among them, the microvascular complications include diabetic nephropathy (DN), diabetic peripheral neuropathy (DPN) and diabetic retinopathy (DR), while the macrovascular complications include cardiovascular diseases (CVDs), stroke and peripheral vascular diseases, such as diabetic foot disease (DFD) (2). Chronic kidney disease (CKD) is a worldwide public health challenge and was first described as a advancing disease that begins with a small amount of albumin in the urine, accompanied with a decrease in kidney function, renal injury, and finally end-stage renal disease (ESRD) (3). CKD is diagnosed by the persistently increased urinary albumin excretion, low estimated glomerular filtration rate (eGFR), or additional characteristics of renal injury $(4,5)$. It has been reported that $\mathrm{DN}$ has become the main cause of CKD in China (6). The rapidly elevating prevalence of diabetes global actually assures that the proportion of CKD due to diabetes, will continue to increase, so called diabetic kidney disease (DKD), also known as DN (7). According to World Health Organization data, about $25-40 \%$ of T2DM patients develop renal injury and CKD, which significantly worsens the quality of life of patients (8). Once disease progresses, about $20 \%$ to $40 \%$ of patients irreversibly would develop ESRD (9).

DKD is one of the usual complications of T2DM and is the major cause of ESRD, CVDs and death, but it is difficult to determine which T2DM patients have a higher risk of DKD based solely on glomerular filtration rate and proteinuria. Therefore, there is an urgent need to find biomarkers that can identify high-risk populations of DKD early in diabetes, through which we can predict the disease and prevent the progression of the disease at the early stage.

Metabolomics, a rapidly developing field in systems biology, measures metabolic changes in response to the development and progress of the disease $(10,11)$. It has been widely used to detect metabolic profiles of diabetesrelated diseases and to identify potential biomarkers (12). Rossi et al. (13) used liquid chromatography coupled to tandem mass spectrometry (LC-MS/MS) to measure whole blood from streptozotocin-induced DN with tissue inhibitors of metalloproteinase 3-deficient mice, and reported that the abnormal fatty acid metabolism and the related elevations in serum acyl-carnitines (ACs) were associated with the pathogenic process of DN. Li et al. (14) used gas chromatograph coupled with time-offlight mass spectrometry (GC-TOFMS) to determine the urinary metabolites acquired from $\mathrm{DN}$ patients and showed that reduced level of mitochondrial metabolites were closely related to the occurrence of albuminuria. Our former study showed that serum differential metabolites, such as carbohydrates, fatty acids, amino acids, bile acids, lipids, steroids, and tricarboxylic acids, were involved in the formation of T2DM complications according to a 292-patient cohort study using untargeted metabolomics (15). We also recently summarized many different metabolomics studies on diabetic complications (16). As far as we known, no large-scale metabolomics research has characterized serum and urine metabolites systemically, especially in DN.

In this research, we performed serum and urine metabolic profiling between T2DM patients with or without nephropathy using GC-TOFMS. We also analyzed the characteristics of serum and urine metabolites in patients at different stages of DN to investigate the association between serum and urine metabolites and during the development of DN. The purpose of this research was to probe the metabolite changes at different stages of $\mathrm{DN}$, to define the metabolites that are potentially predictive markers for $\mathrm{DN}$.

\section{Methods}

\section{Chemicals and reagents}

Methoxyamine $\mathrm{HCl}$, fatty acid methyl ester (C7-C30, FAMEs) standards, pyridine and anhydrous sodium sulfate were purchased from Sigma-Aldrich (St. Louis, MO, USA). N-methyl-N-trimethylsilyl trifluoroacetamide (MSTFA) with 1\% (vol/vol) trimethylchlorosilane (TMCS), acetonitrile, methanol, dichloromethane, hexane, chloroform and acetone were obtained from ThermoFisher Scientific (FairLawn, NJ, USA). Ultrapure water was produced by a Mill-Q Reference system equipped with an LCMS Pak filter (Millipore, Billerica, MA).

\section{Study population}

From March 2015 to March 2016, T2DM patients were collected from the endocrinology clinics of Shuguang 
Hospital, Putuo Hospital and Shanghai Traditional Chinese Medicine Hospital affiliated to Shanghai University of Traditional Chinese Medicine. The 2010 diagnostic criteria from the American Diabetes Association (ADA) were used: (I) glycosylated hemoglobin (HbA1c) $\geq 6.5 \%$, (II) fasting plasma glucose $(\mathrm{FPG}) \geq 7.0 \mathrm{mmol} / \mathrm{L}$ (fasting is defined as at least 8 hours without calorie); (III) 2 hours of blood glucose during oral glucose tolerance analysis $\geq 11.1 \mathrm{mmol} / \mathrm{L}$, and (IV) in a typical hyperglycemia or hyperglycemia crisis patients, random blood glucose $\geq 11.1 \mathrm{mmol} / \mathrm{L}$ (17). DN was diagnosed as diabetes with proteinuria (the value ratio of urinary albumin to creatinine $\geq 30 \mathrm{mg} / \mathrm{g}$ ), damaged glomerular filtration rate (GFR) $\left[<60 \mathrm{~mL} / \mathrm{min} / 1.73 \mathrm{~m}^{2}\right.$ evaluated using the chronic kidney disease epidemiology collaboration (CKD-EPI) equation], or both (18). For women with serum creatinine $\leq 0.7$, GFR was calculated as $144 \times$ (serum creatinine/ 0.7$)^{-0.329} \times(0.993)^{\text {age }}$; for those who $>0.7$, GFR was computed as $144 \times$ (serum creatinine/0.7 $)^{-1.209}$ $\times(0.993)^{\text {age }}$; for men with serum creatinine $\leq 0.9$, GFR was computed as $141 \times(\text { serum creatinine } / 0.9)^{-0.411} \times(0.993)^{\text {age }}$; and for those who $>0.9$, GFR was computed as $141 \times$ (serum creatinine/0.9 $)^{-1.209} \times(0.993)^{\text {age }}(19)$. Renal biopsy is the gold standard for distinguishing $\mathrm{DN}$ and nondiabetic nephropathy, but renal biopsy is an invasive test and the patient's acceptance is low (20,21). The exclusion criteria were as follows: (I) primary kidney disease, this experiment is based on the diagnosis of diabetes, proteinuria, diabetic retinopathy, hematuria, and systolic blood pressure. (II) urinary tract infection, (III) recent acute disease, (IV) malignant tumor, (V) taking drugs affecting bone metabolism in the past six months, and (VI) primary osteoporosis and other endocrine diseases, autoimmune diseases, or similar conditions. Those who were difficult to accurately identify kidney disease was also excluded in this study. Additionally, hypertensive patients were excluded based on their medical histories and measured blood pressure. The information about weight, height, waist-tohip ratio (WHR) and body mass index (BMI) was collected. All patients who participated in the study offered a signed informed consent form. The ethical approval for the present study was provided by the Ethics Committee of Shanghai University of Traditional Chinese Medicine.

\section{GC-TOFMS analysis}

All serum and urine samples were dissolved on ice and centrifuged at $4{ }^{\circ} \mathrm{C}$ and $3,000 \mathrm{~g}$ for $5 \mathrm{~min}$ (Microfuge 20R, Beckman Coulter, Inc., Indianapolis, IN, USA) to separate debris or a lipid layer. Each $50 \mu \mathrm{L}$ of serum sample aliquot was mixed with $10 \mu \mathrm{L}$ of the internal standard, to which $175 \mu \mathrm{L}$ of prechilled methanol/chloroform $(\mathrm{v} / \mathrm{v}=3 / 1)$ was added. After mixed at $-20^{\circ} \mathrm{C}$ for $20 \mathrm{~min}$ and centrifuged at $14,000 \mathrm{~g}$ and $4{ }^{\circ} \mathrm{C}$ for $20 \mathrm{~min}$, the supernatant was transferred to an autosampler vial (Agilent Technologies, Foster City, CA, USA) carefully. In order to remove chloroform, all samples in autosampler vials were evaporated using a CentriVap vacuum concentrator (Labconco, Kansas City, MO, USA). Each urine sample aliquot of $75 \mu \mathrm{L}$ was mixed with $10 \mu \mathrm{L}$ of internal standard and was further lyophilized with a FreeZone dryer equipped with a stopping tray dryer (Labconco, Kansas City, MO, USA).

The samples were further analyzed by a GC-TOFMS system (Pegasus HT, Leco Corp., St. Joseph, MO, USA) coupled to an Agilent 7890B gas chromatograph and a Gerstel multipurpose MPS2 sampler with dual heads (Gerstel, Muehlheim, Germany). An Rxi-5 ms capillary column was used for separation $(30 \mathrm{~m} \times 250 \mu \mathrm{m}, 0.25-\mu \mathrm{m}$ film thickness; Restek Corporation, Bellefonte, PA, USA). The carrier gas was set at a constant flow rate of $1.0 \mathrm{~mL} / \mathrm{min}$ using helium. Injection and transfer interface temperatures were both set to $270{ }^{\circ} \mathrm{C}$, and source temperature was $220^{\circ} \mathrm{C}$. The analysis was made using electron impact ionization $(70 \mathrm{eV})$ in the full scan mode ( $\mathrm{m} / \mathrm{z}$ 50-500).

\section{Statistical analysis}

SPSS version 19.0 (SPSS Inc., Chicago, IL, USA) was used for analysis, and $\mathrm{P}<0.05$ was considered statistically significant. Data are presented as the medians (interquartile ranges, IQRs). T-test, Wilcoxon test and Analysis of Variance (ANOVA) test were performed between two groups or among multiple groups. Chi-square tests were used to compare categorical variables. Partial least squaresdiscriminant analysis (PLS-DA) and orthogonal partial least squares-discriminant analysis (OPLS-DA) were performed using SIMCA-P + 12.0 (Umetrics AB).

\section{Results}

Clinical information of T2DM patients with or without nephropathy

The clinical characteristics of the 88 subjects, including $44 \mathrm{DN}$ and 44 T2DM subjects, are summarized in Table 1. According to clinical and pathological processes, the 


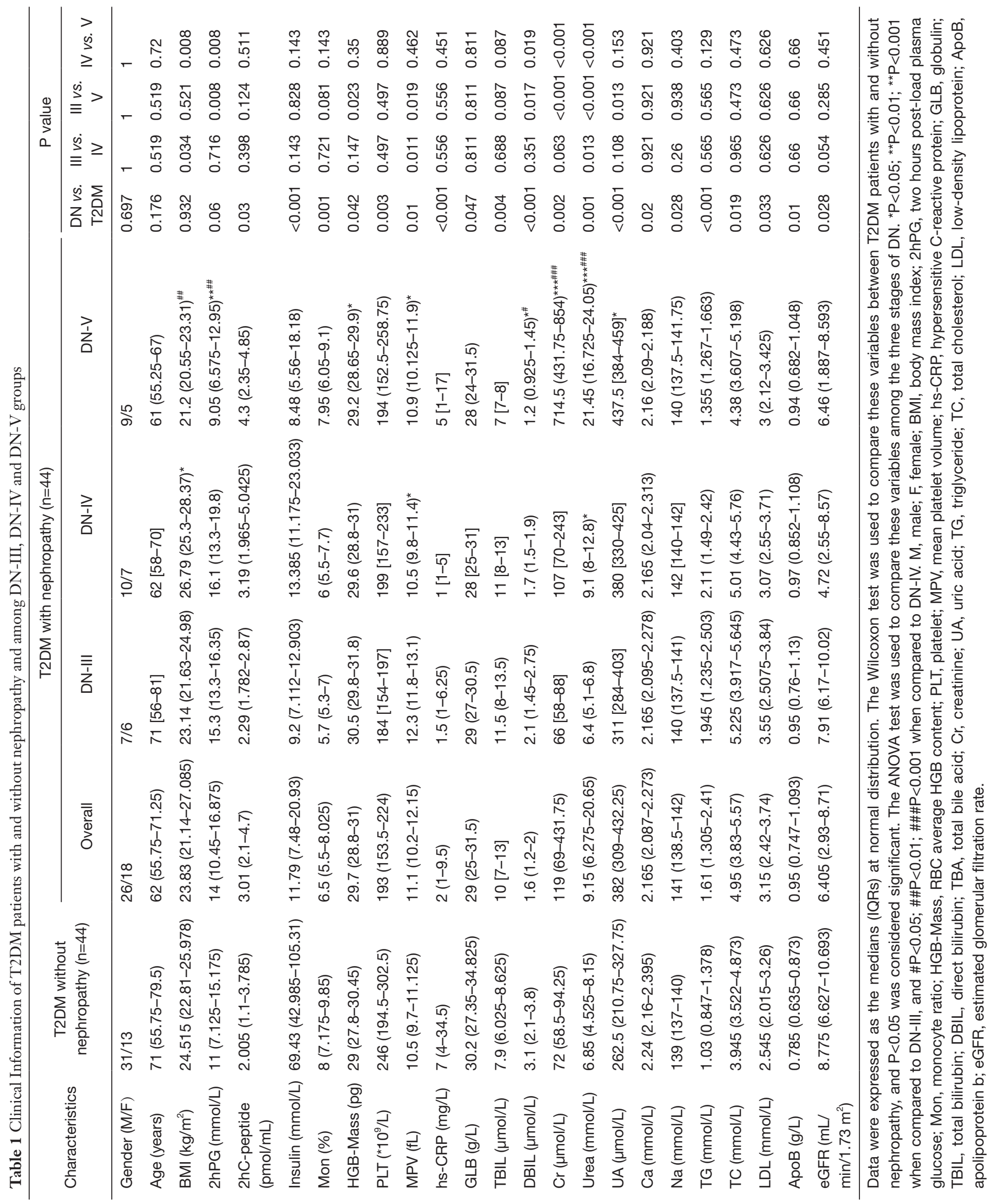


Mogensen staging method divides DN into 5 phases: glomerular hyperfiltration and renal hypertrophy (DN-I), normal albuminuria (DN-II), early diabetic nephropathy (DN-III), clinical diabetic nephropathy (DN-IV), and endstage renal failure (DN-V) (22). At present, the clinical diagnosis of $\mathrm{DN}$ is mainly based on microalbuminuria, while patients with stage I and II DN have no good diagnostic signs, making the condition difficult to diagnose and intervene early. In the present study, according to the Mogensen staging criteria, patients with $\mathrm{DN}$ were further divided into three stages: DN-III ( $\mathrm{n}=13), \mathrm{DN}-\mathrm{IV}(\mathrm{n}=17)$ and $\mathrm{DN}-\mathrm{V}(\mathrm{n}=14)$.

This study showed that the average T2DM and DN patients gender, age and BMI had no significant differences between the T2DM and DN patients $(\mathrm{P}>0.05)$ (Table 1). Compared with the T2DM group, the serum insulin, monocyte ratio, platelet (PLT), total bilirubin (TBIL), direct bilirubin (DBIL), hypersensitive C-reactive protein (hs-CRP), creatinine (Cr), uric acid (UA) and triglyceride (TG) levels of DN patients showed significant differences $(\mathrm{P}<0.01)$. Accompanied with the progression of the disease, the serum urea level increased gradually $(\mathrm{P}<0.05)$, indicating that the serum urea level may be a potential clinical indicator for the diagnosis of whether T2DM progresses into DN or the severity of DN. The clinical features of other T2DM patients with or without nephropathy are detailed in Table S1.

\section{GC-TOF-MS data analysis of serum and urine in T2DM patients with or without nephropathy}

We used GC-TOFMS metabolomics to analyze the serum and urine metabolites of T2DM patients with or without nephropathy. A total of 177 serum metabolites and 159 urine metabolites were determined including amino acids, carbohydrates, fatty acids and organic acids. In order to determine if serum and urine metabolites differed between T2DM patients with or without nephropathy, we constructed OPLS-DA models, which have been broadly used in metabolomics researches $(23,24)$. Typically, R2Y provides the fitting degree of the model to the $\mathrm{Y}$ data, while Q2Y is used to estimate the fitting degree of the model to Y. To obtain high predictive ability, Q2Y and R2Y values should be close to 1 . As shown in Figure 1A,B, there is a distinct difference in clustering of between DN and T2DM groups. The R2Y and Q2Y were 0.87, 0.822 and 0.797, 0.71 in serum and urine models, respectively, indicating that the models had fine prediction features. The 20- time permutation test showed that the model was steady and reliable (25), with cumulative Q2 at -0.441/-0.265 (Figure S1). As shown in Figure 1C,D, we also obtained differential metabolites by Student's T-test for serum and urine. In Figure 1C, the $\mathrm{P}$ values and $\log 1.5$ (fold change, FC) are shown, with cut-off values of $0.05,0.01$ for $\mathrm{P}$ value and 1.5 for log.1.5 FC, respectively. The highlighted serum metabolites from the top right corner are positively correlated with subjects on the right side of the OPLS-DA score plot (Figure 1A). The highlighted serum metabolites from the top left corner are negatively correlated with subjects on the right side of the OPLS-DA score plot (Figure $1 A$ ), indicating that these metabolites are decreased in these subjects compared to those on the left side of the OPLS-DA score plot. In Figure 1D, the P values and log 1.5 FC are introduced with cut-off values of 0.05 for $\mathrm{P}$ value and 1.5 for log.1.5 FC, respectively. The highlighted urinary metabolites from the top right corner are positively correlated with subjects on the right side of the OPLS-DA score plot (Figure 1B). The highlighted urine metabolites from the top left corner are negatively correlated with subjects located on the right side of the OPLS-DA score plot, indicating that these metabolites are decreased in these subjects compared to those on the left side of the OPLSDA score plot (Figure 1B).

\section{Metabolic features in serum and urine of T2DM patients with or without nephropathy}

In order to select potential biomarkers associated with $\mathrm{DN}$, independent sample T-test was performed for the variables between DN and T2DM group, and the differences were statistically significant. Significant differences in metabolites between the two groups $(\mathrm{P}<0.05)$ were recognized as potential biomarkers. Then a variable importance plot (VIP) was applied to screen out the important metabolites in the model. In the present research, metabolites with VIP $>1$ were recognized as candidate biomarkers. According to the above criteria, 61 serum metabolites and 46 urine metabolites were filtered as candidate biomarkers for DN. The summary of the serum and urine biomarker identified in DN is shown in Tables 2 and 3, respectively. More complete information is shown in Supplementary Tables $S 2$ and $S 3$. Among them, the nine most significant changes in serum metabolites are shown in Figure $2 \mathrm{~A}$ $(\mathrm{P}<5 \mathrm{E}-06$ and VIP $\geq 1.5)$. Compared with T2DM patients, benzoic acid, fumaric acid, erythrose, and L-Arabitol in the DN group were significantly increased. In contrast, 
A

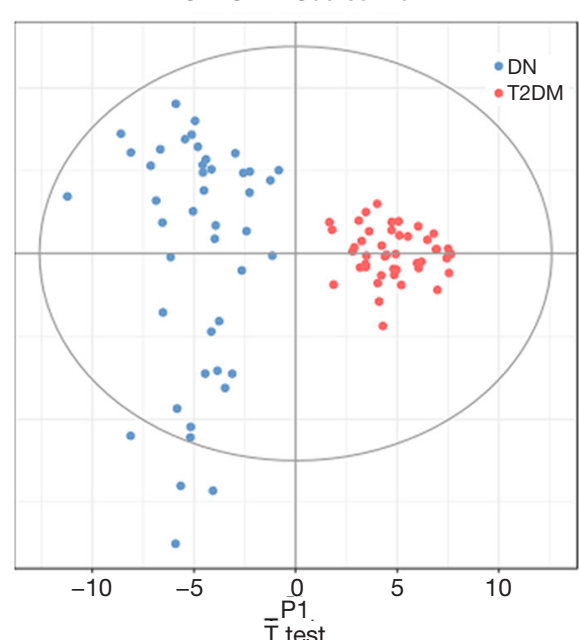

C

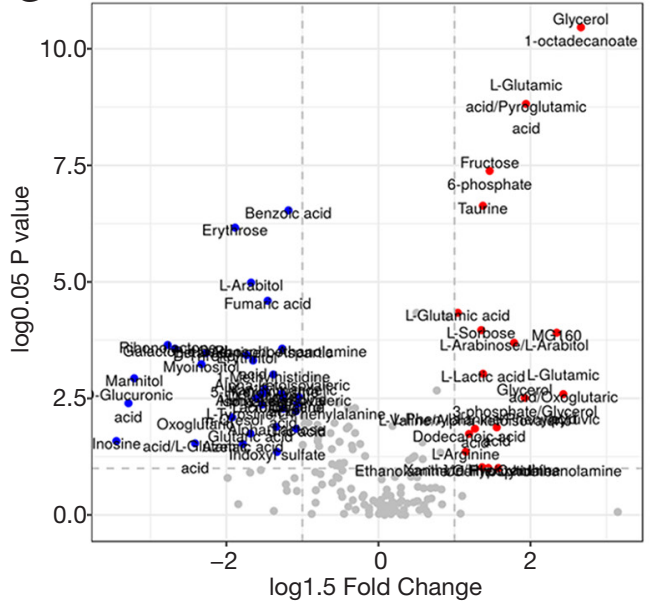

B

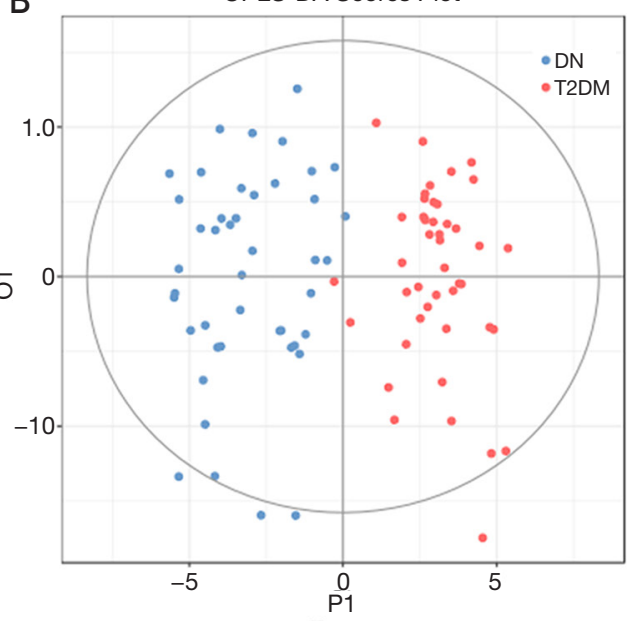

D

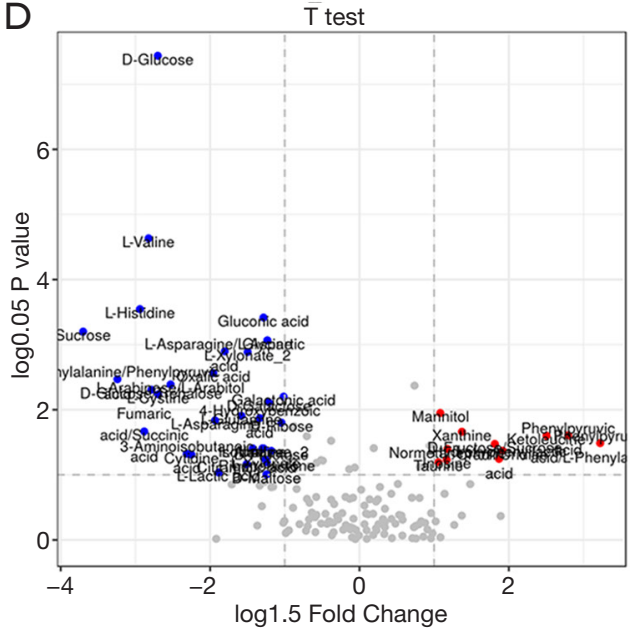

Figure 1 Visualization of overall serum and urinary metabolite profile differences between the two groups. (A) OPLS-DA score plot of serum. (B) OPLS-DA score plot of urine. T2DM with nephropathy group (blue), T2DM without nephropathy group (red). (C) Volcano plots of differentially expressed serum metabolites in T2DM patients with or without nephropathy. X-axis, $\log 1.5$ (fold change); Y-axis, $\log 0.05$ (P value). (D) Volcano plots of differentially expressed urinary metabolites in T2DM patients with or without nephropathy. X-axis, $\log 1.5$ (fold change); Y-axis, $\log 0.05$ ( $\mathrm{P}$ value). The red highlighted points from the right $(\mathrm{P}<0.05, \mathrm{FC}>1)$ indicated that these metabolites are significantly increased. The blue highlighted points from the left $(\mathrm{P}<0.05, \mathrm{FC}<1)$ indicate that these metabolites are significantly decreased. The noncolored points indicate that these metabolites are not significant.

glycerol 1-octadecanoate, L-glutamic acid/pyroglutamic acid, fructose 6-phophate, taurine and L-glutamine in DN patients were significantly decreased. The 9 most significant changes in urinary metabolites are shown in Figure $2 B(\mathrm{P}<5 \mathrm{E}-04$ and VIP $\geq 1.5)$. Compared with $\mathrm{T} 2 \mathrm{DM}$ patients, urine levels of $\mathrm{D}$-glucose, L-valine, L-histidine, sucrose, gluconic acid, glycine, L-asparagine/L-aspartic acid, L-xylonate-2, and oxalic acid in the DN group were significantly increased.

In order to determine which metabolic pathways were major influenced by $\mathrm{DN}$, we summarized pathways analysis based on $\mathrm{P}$ values from their impact values and enrichment. As shown in Figure S2, the "metabolome view", on the based of enrichment analysis (Y-axis) and topology analysis (X-axis), the arrangement shows all metabolic pathways, in which red presents the most significant $\mathrm{P}$ values, while yellow and white represent the lowest $\mathrm{P}$ values. In order to know the biological significance of the metabolic changes, we conducted a functional enrichment analysis of the experimental data using MetaboAnalyst (26), which provides metabolite set enrichment analysis (MSEA) for metabolites of serum and urine. Then, the metabolic pathways with 


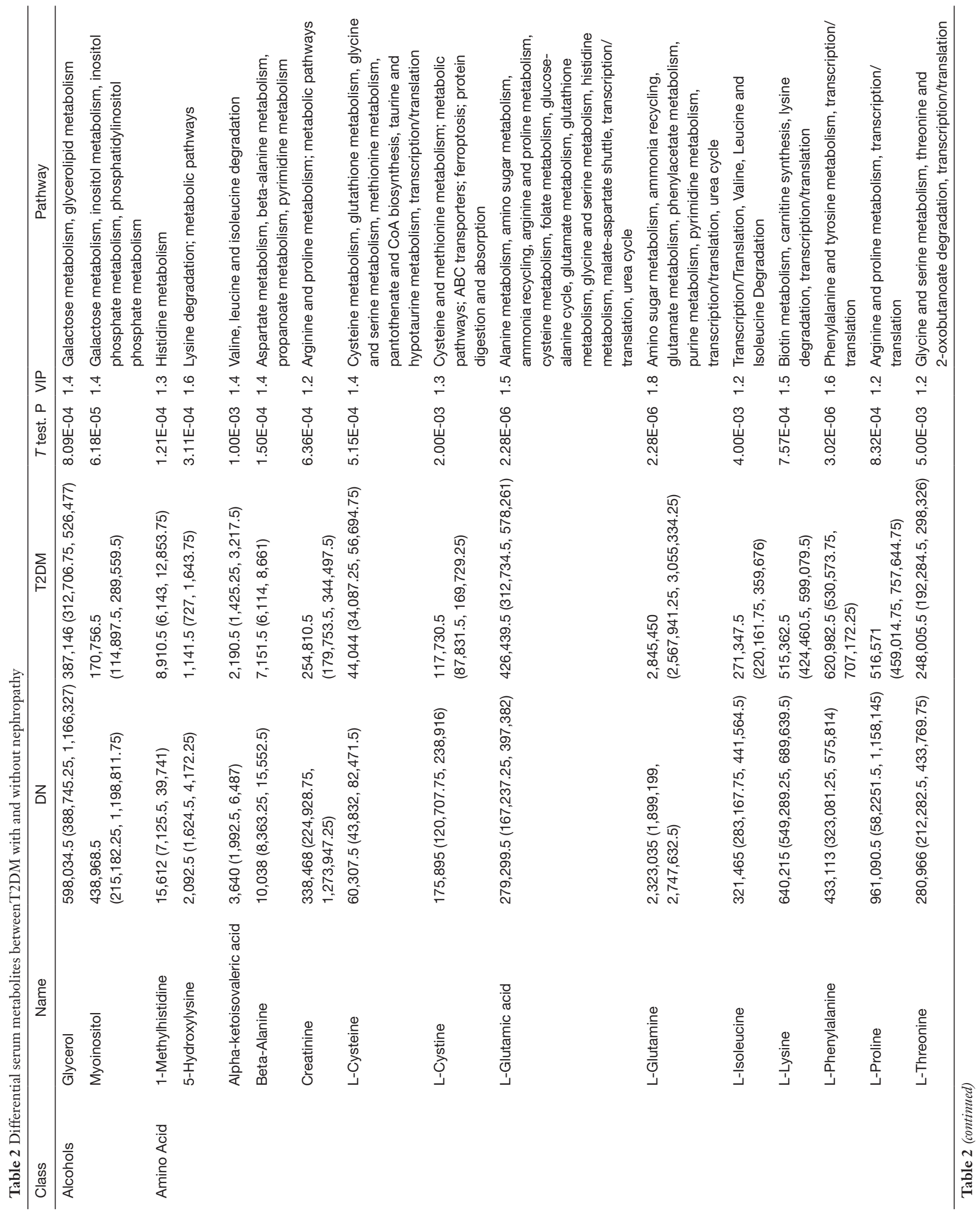




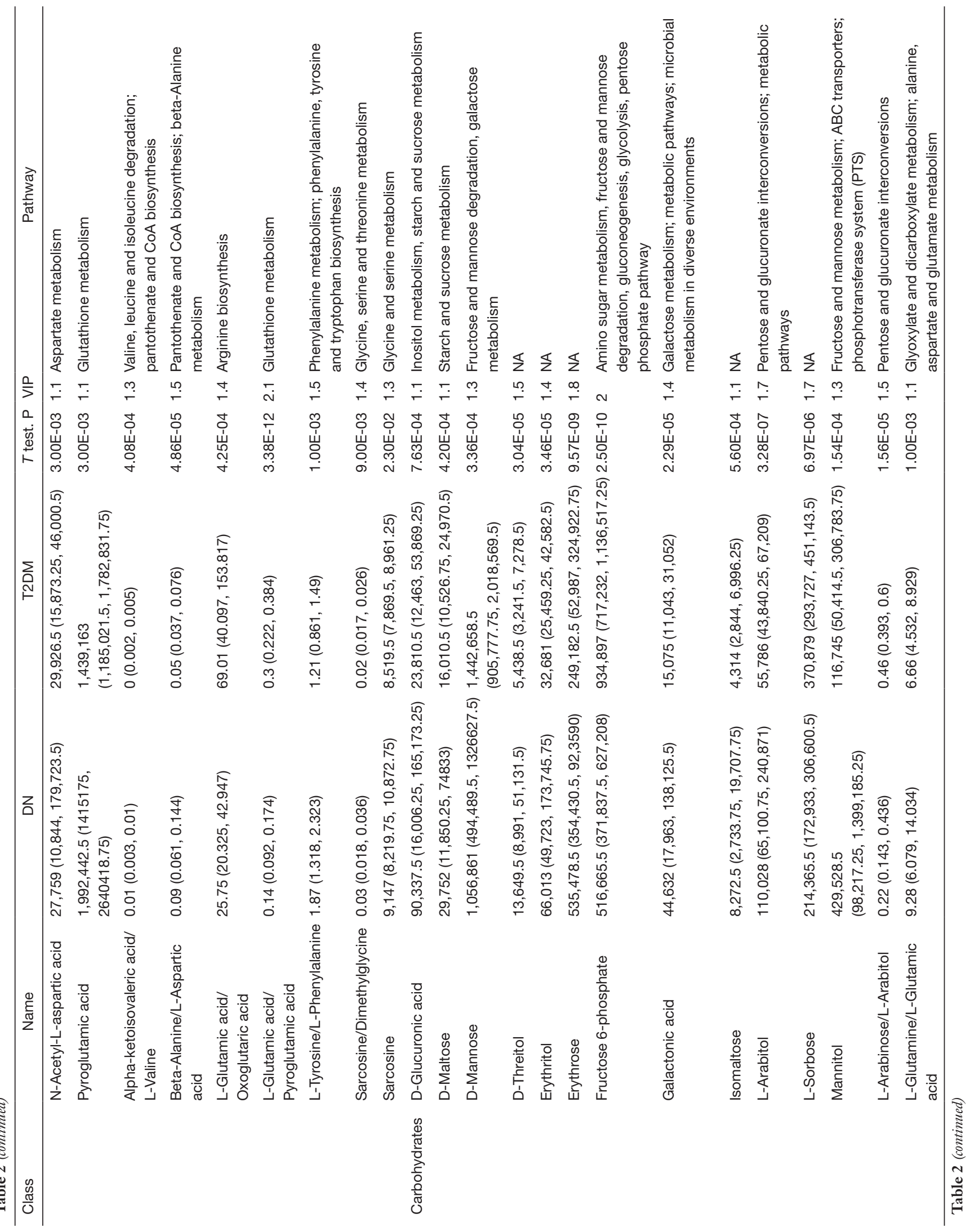




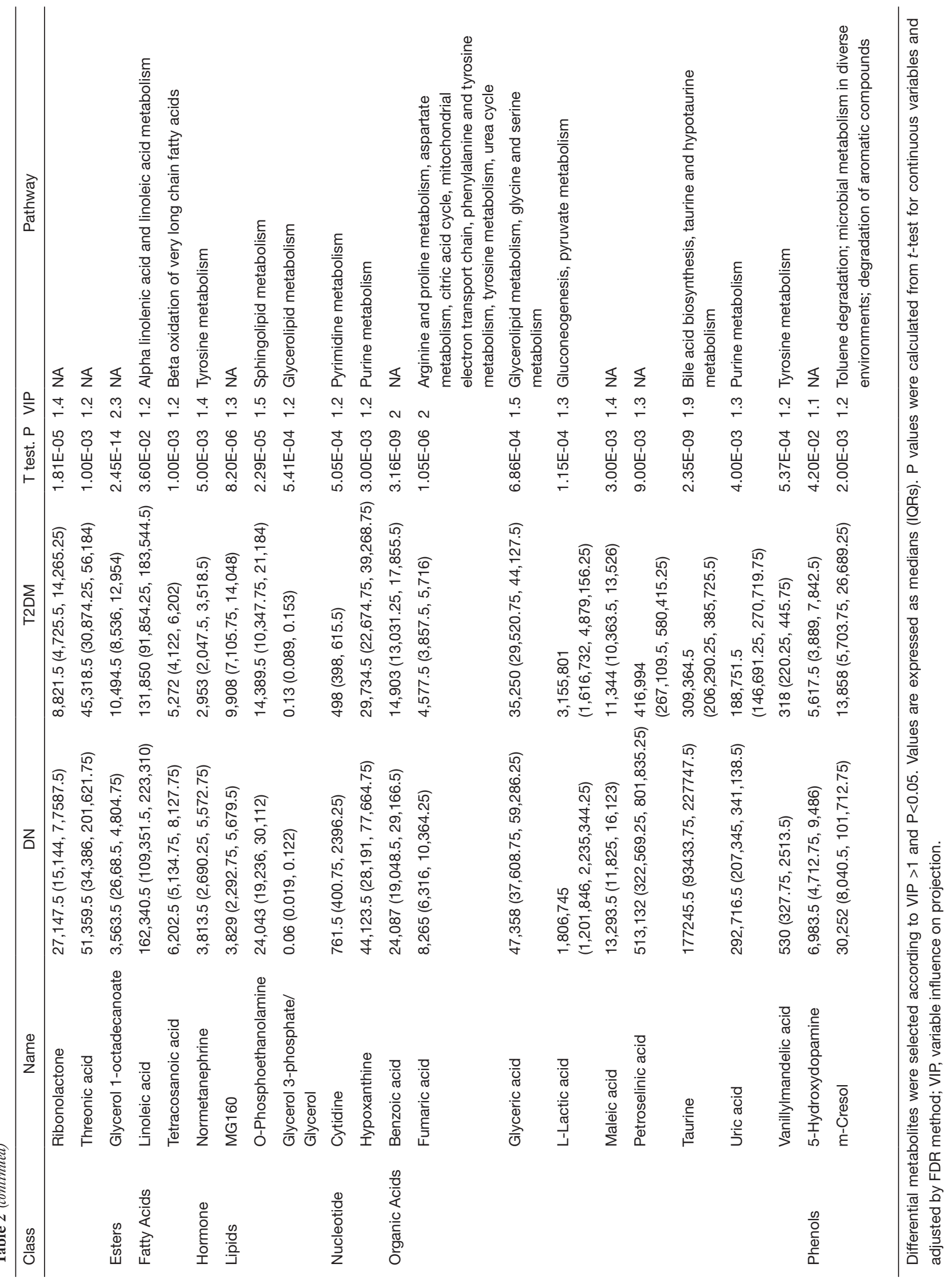




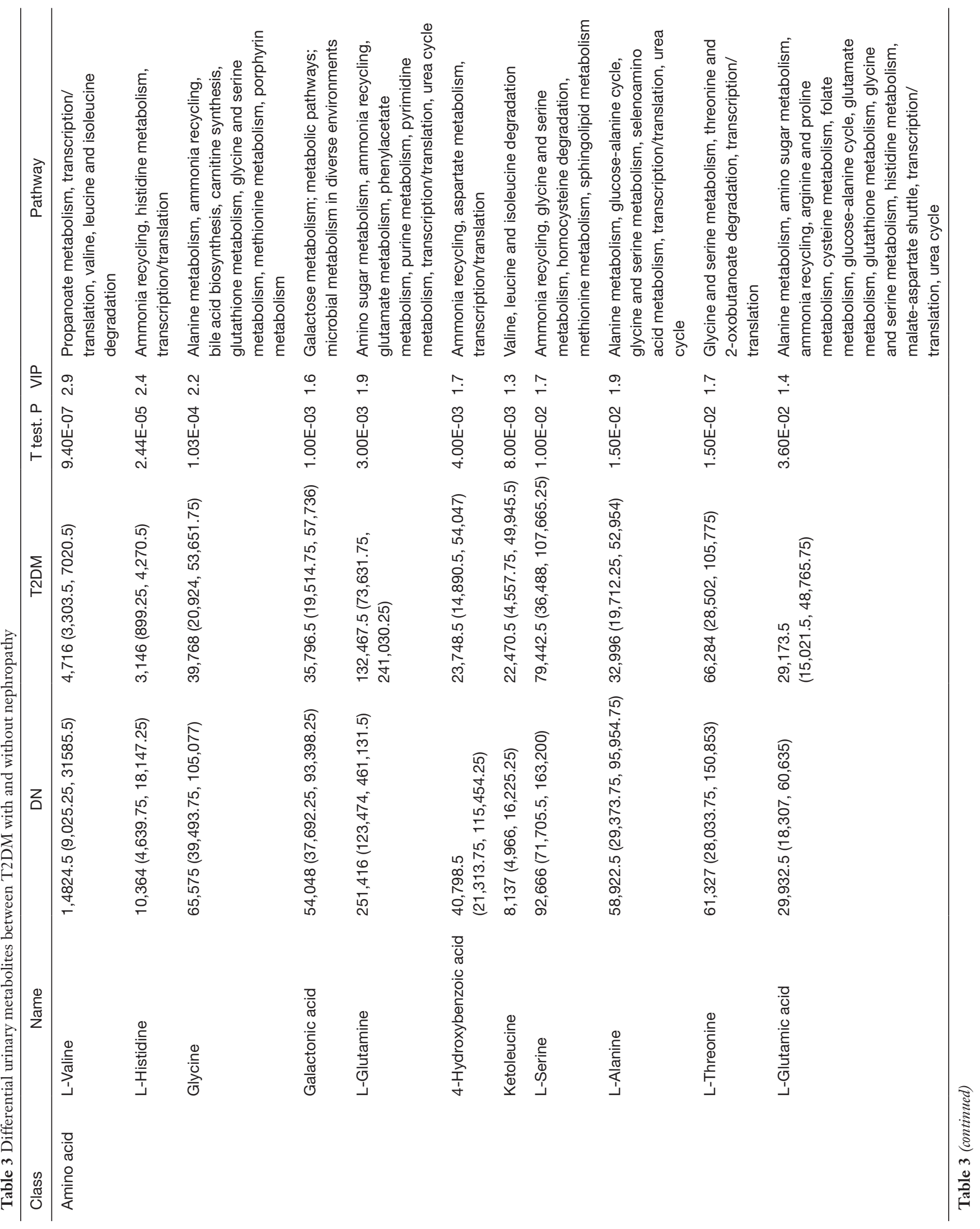




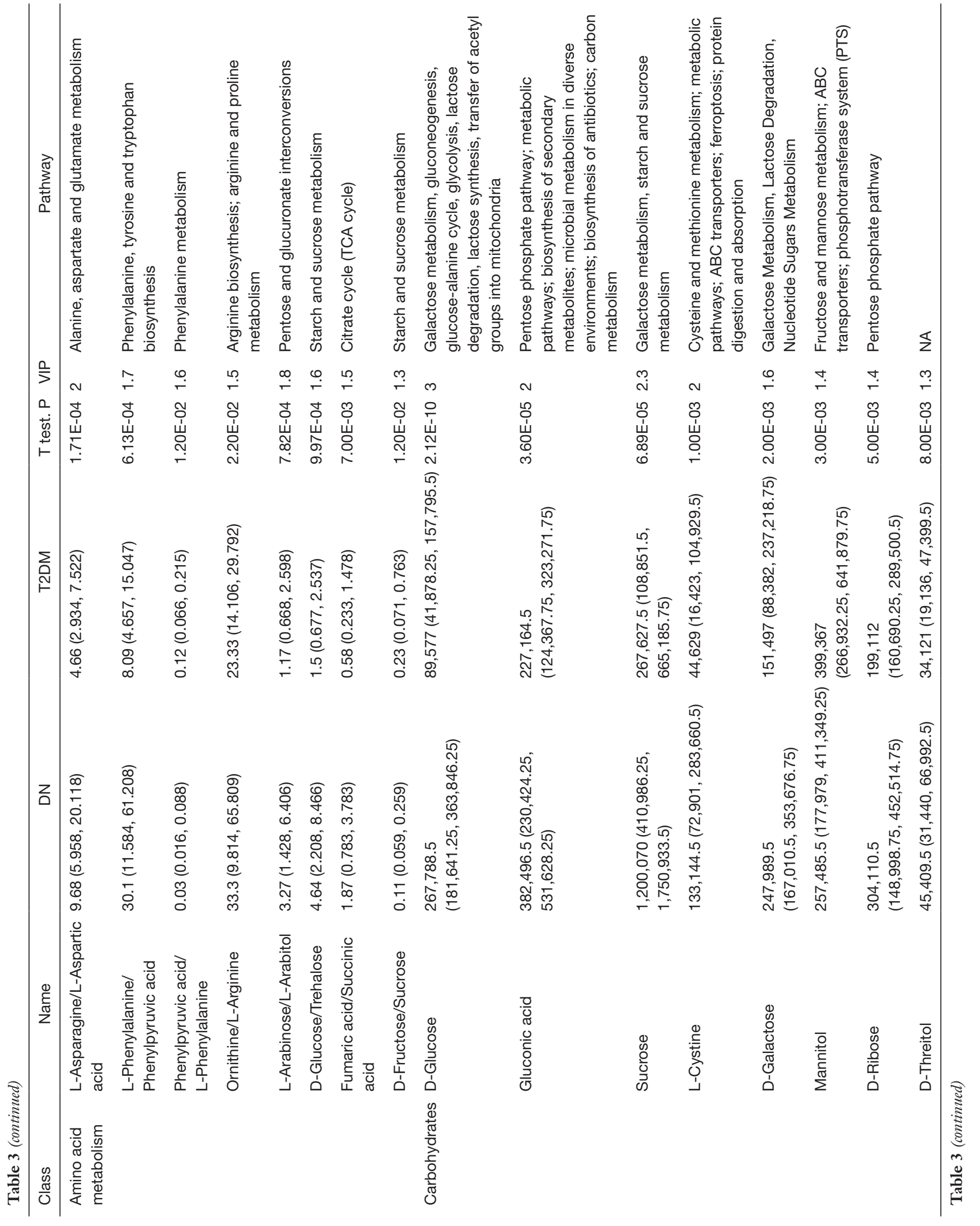




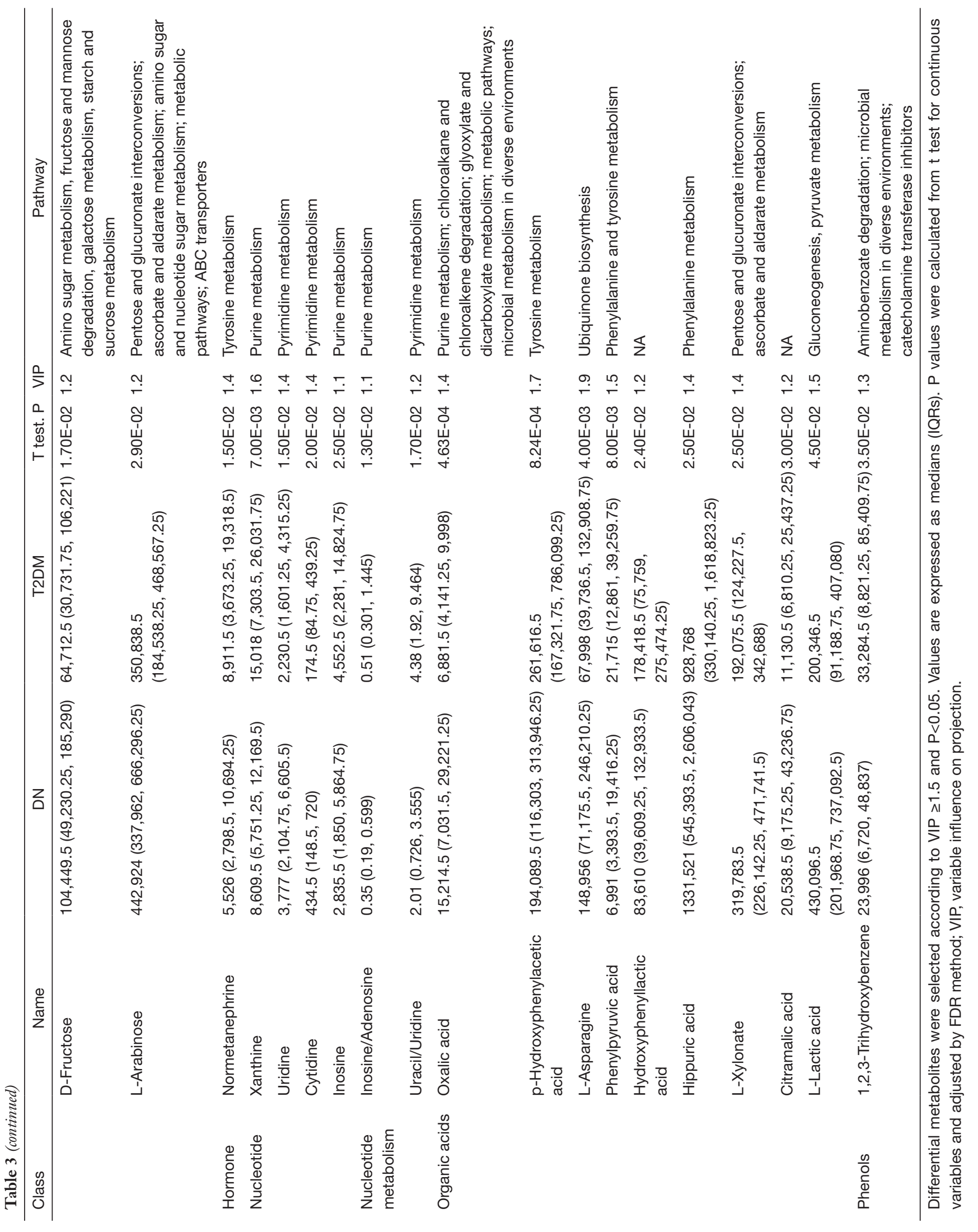


A

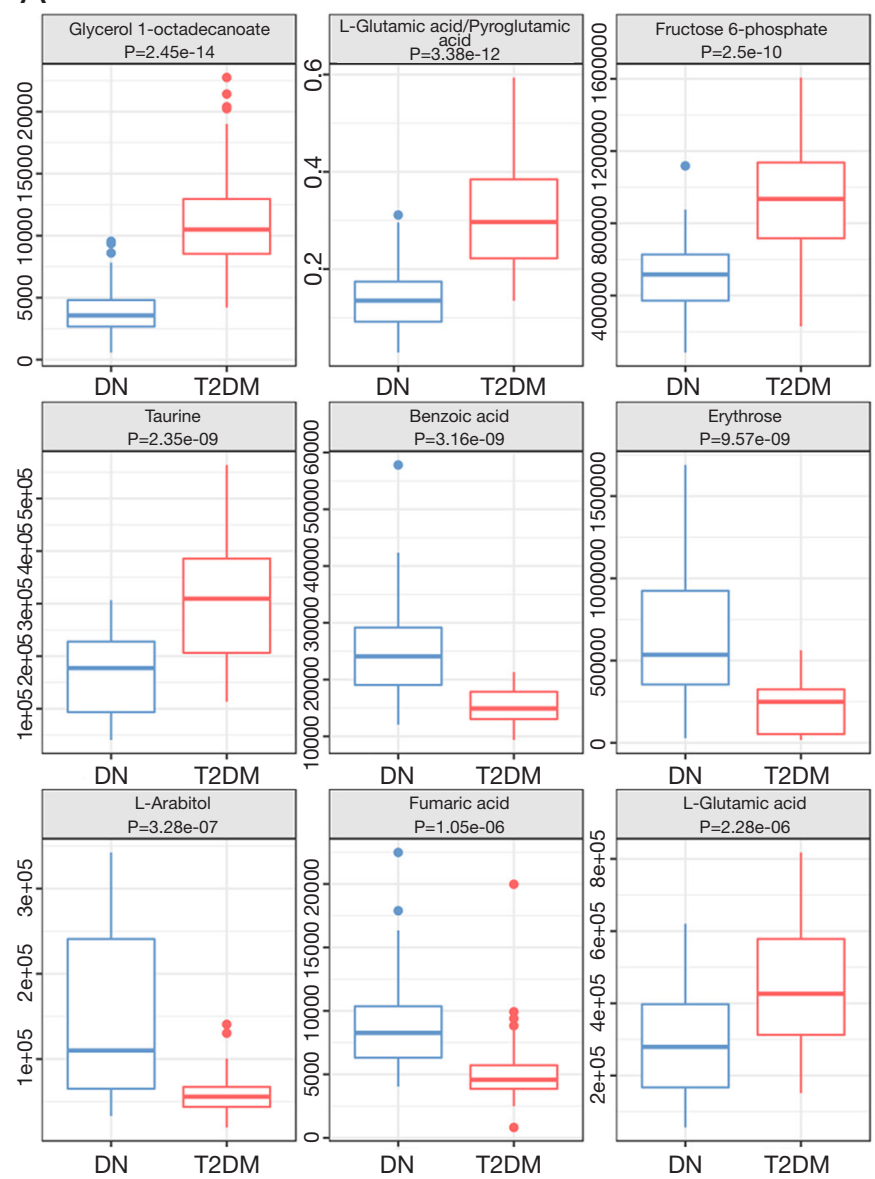

B

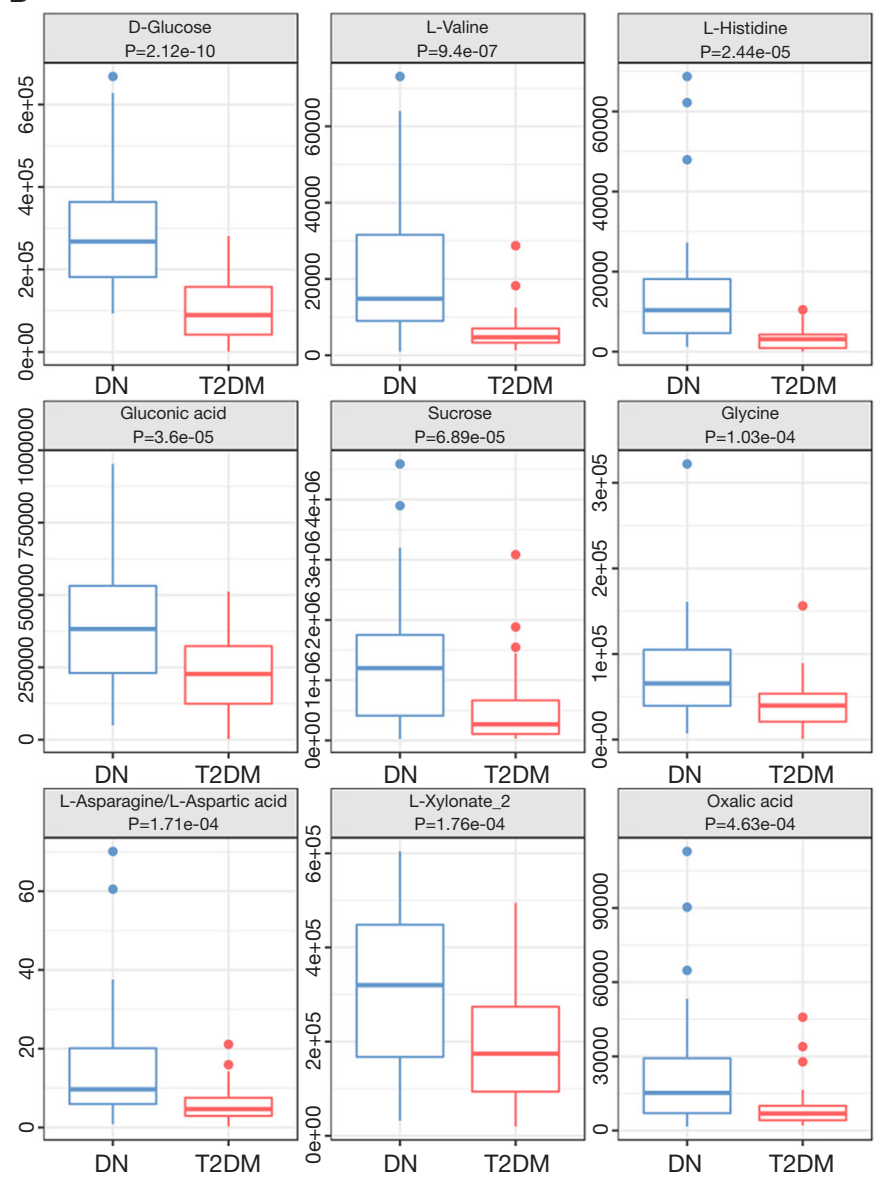

Figure 2 Top ranked differential serum and urinary metabolites between the two groups. (A) The peak height comparison of representative serum metabolites in two groups, T2DM with nephropathy group (blue), T2DM without nephropathy group (red), whose VIP value $>1.5, \mathrm{P}<5 \mathrm{E}-06$. The statistical analysis of the significance between T2DM with nephropathy and without nephropathy group. Glycerol 1-octadecanoate, $\mathrm{P}=2.45 \mathrm{E}-14$. L-glutamic acid/pyroglutamic acid, $\mathrm{P}=3.38 \mathrm{E}-12$. Fructose 6-phophate, $\mathrm{P}=2.5 \mathrm{E}-10$. Taurine, $\mathrm{P}=2.35 \mathrm{E}-09$. Benzoic acid, P=3.16E-09. Erythrose, P=9.57E-09. L-Arabitol, P=3.28E-07. Fumaric acid, $\mathrm{P}=1.05 \mathrm{E}-06$. L-Glutamine, $\mathrm{P}=2.28 \mathrm{E}-06$. (B) The peak height comparison of representative urinary metabolites in two groups, T2DM with nephropathy group (blue) = T2DM without nephropathy group $(\mathrm{red})=$ whose VIP value $>1.5, \mathrm{P}<5 \mathrm{E}-04$. The statistical analysis of the significance between T2DM with nephropathy group and T2DM without nephropathy group. D-Glucose, $\mathrm{P}=2.12 \mathrm{E}-10$. L-Valine, $\mathrm{P}=9.4 \mathrm{E}-07$. L-Histidine, $\mathrm{P}=2.44 \mathrm{E}-05$. Gluconic acid, $\mathrm{P}=3.6 \mathrm{E}-05$. Sucrose, $\mathrm{P}=6.89 \mathrm{E}-05$. Glycine, $\mathrm{P}=1.03 \mathrm{E}-04$. L-Asparagine/L-aspartic acid, $\mathrm{P}=1.71 \mathrm{E}-04$. L-Xylonate-2, $\mathrm{P}=1.76 \mathrm{E}-04$. Oxalic acid, $\mathrm{P}=4.63 \mathrm{E}-04$.

significant $\mathrm{P}$ values $<0.05$ were screened out (Table 4). Finally, 9 and 12 metabolic pathways were identified with significant differences in serum and urine metabolism, respectively. As the Table 4 shows, 9 metabolic pathways are closely related to the occurrence of serum metabolism of $\mathrm{DN}$, including arginine biosynthesis, galactose metabolism, valine, leucine and isoleucine biosynthesis, starch and sucrose metabolism, alanine, aspartate and glutamate metabolism, glutathione metabolism, D-glutamine and D-glutamate metabolism, glycine, serine and threonine metabolism, and nitrogen metabolism. Additionally, 12 metabolic pathways are closely related to the occurrence of urinary metabolism of DN, including starch and sucrose metabolism, valine, leucine, isoleucine biosynthesis, alanine, aspartate and glutamate metabolism, phenylalanine, tyrosine and tryptophan biosynthesis, glyoxylate and dicarboxylate metabolism, phenylalanine metabolism; glycine, serine and threonine metabolism, D-glutamine and D-glutamate metabolism, arginine biosynthesis, nitrogen metabolism, galactose metabolism, and histidine metabolism. The 
Table 4 Top-ranked altered serum and urine metabolic pathways

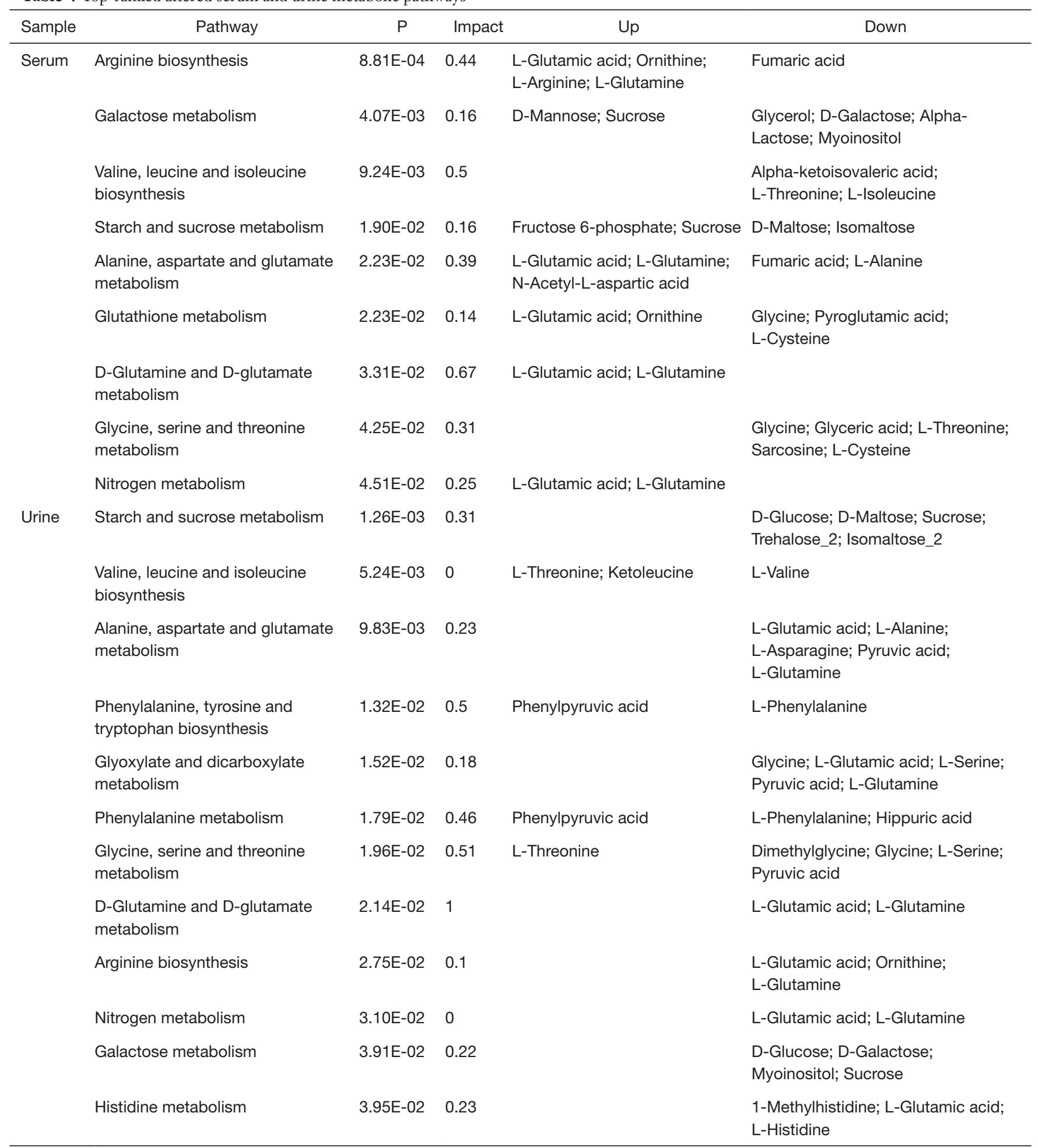


significant serum and urinary metabolites observed in this study are shown in Figure 3.

\section{Serum and urinary metabolic profiles in T2DM patients with nephropathy at different stages}

We further compared the serum metabolites in three groups of patients with different stages of $\mathrm{DN}$, and the score plots of PLS-DA are shown in Figure $4 A$. According to the model validation results, the PLS-DA $(\mathrm{R} 2 \mathrm{X}=0.21$; $\mathrm{R} 2 \mathrm{Y}=0.326$; $\mathrm{Q} 2 \mathrm{Y}=0.29$ ) showed distinct separation among all three groups. A 999-time permutation test was used to validate the reliability of the model, as illustrated in Figure $4 B$, with R2 =0.132 and Q2 =-0.105. Based on the VIP value of the PLS-DA (VIP >1), combined with ANOVA, 28 potential biomarkers were ultimately identified $(\mathrm{P}<0.05)$, including two species of organic acids (vanillylmandelic acid and taurine), ten carbohydrates, six amino acids and other metabolites (Table 5).

We also compared the urinary metabolites of the three groups of patients in different DN stages, and the PLS-DA score plot is shown in Figure 4C. PLS-DA model [R2X (cum) $=0.09 ; \mathrm{R} 2 \mathrm{Y}(\mathrm{cum})=0.297 ; \mathrm{Q} 2 \mathrm{Y}=0.118]$ indicated good separation among all three groups. A 999-time permutation test was used to validate the reliability of the model, as illustrated in Figure 4D, with R2 $=0.172$ and Q2 $=-0.079$. Based on the VIP value of the PLS-DA (VIP >1), combined with ANOVA, 5 potential biomarkers were ultimately identified $(\mathrm{P}<0.05)$, including one organic acid, two types of carbohydrates (galactonic acid and L-arabinose), and their metabolites (Table 5).

\section{Discussion}

$\mathrm{DN}$ is a serious complication of T2DM. Patients with DN are at a significantly high risk for ESRD, and further the risks for cardiovascular morbidity and mortality increase persistently $(3,9)$. Thus, the early diagnosis of DN can decrease morbidity by allowing for potential therapeutic interventions. However, there is no single diagnostic marker for the detection of DN. Over the last three decades, a large amount of scientific evidence has shown that many inflammatory markers are related to $\mathrm{DN}$, for instance interleukin-1 (IL-1), IL-8, IL-6, tumor necrosis factoralpha, transforming growth factor beta-1, cytokines, and the neutrophil-lymphocyte ratio in the complete blood count $(27,28)$. Although there have been many studies on the occurrence and development of DN, the overall metabolic changes of T2DM that progress to kidney injury still lack clear characteristics. In this study, we used GCTOFMS to identify distinct metabolic changes and related pathways during the occurrence and the development of $\mathrm{DN}$ in serum and urine samples from patients with DN. Dramatic changes in disease development and progression of DN metabolites could be a potential target for diagnosis or treatment.

Urea is a nitrogen-containing organic end product in protein metabolism, which can remove $80-90 \%$ of nitrogen from the human body (29). Recent studies suggest that it is probable that UA and $\mathrm{Cr}$ are involved in the pathogenesis of DN (30,31). Compared with T2DM patients, we found that serum UA and $\mathrm{Cr}$ levels of DN patients observably increased $(\mathrm{P}<0.05)$. Furthermore, when we compared the clinical features of the three stages of DN, we found that serum urea levels increased as the disease progressed $(\mathrm{P}<0.05)$. There is a obvious relationship between serum urea levels and disease stage, suggesting that DN progression may be associated with abnormal serum urea. However, we found that three different metabolites among the differential metabolites in serum and urine were involved in the metabolic process of the urea cycle. Those metabolites were fumaric acid, L-glutamine, and L-glutamic acid, which were present at significantly higher levels in the serum of DN patients than in T2DM patients, and L-glutamine, L-alanine and L-glutamate, which were present at significantly lower levels in the urine of DN patients, than in T2DM patients, as shown in Table 2. These changes may be a key warning sign, and if ignored, lead to irreversible kidney damage. However, the detailed mechanisms of serum and urine urea metabolism in T2DM DN need further research.

This research used GC-TOFMS metabolomics approach to identify metabolic changes associated with DN patients (32-34). Our data revealed a variety of organic acid metabolism disorders in DN patients, including lower serum taurine levels and higher serum benzoic acid and fumaric acid levels than in T2DM patients without nephropathy.

Taurine is a lesser-known organic acid in mammalian tissue and has multiple effects, such as bile acid conjugation, osmoregulation, viability and prevention of oxidantinduced tissue injury, and supplements that can stimulate prolactin and insulin release $(35,36)$. Lin et al. (37) detected that taurine administration in diabetic rats conspicuously inhibited any further increase in urinary protein excretion. Winiarska et al. (38) found that taurine had the 

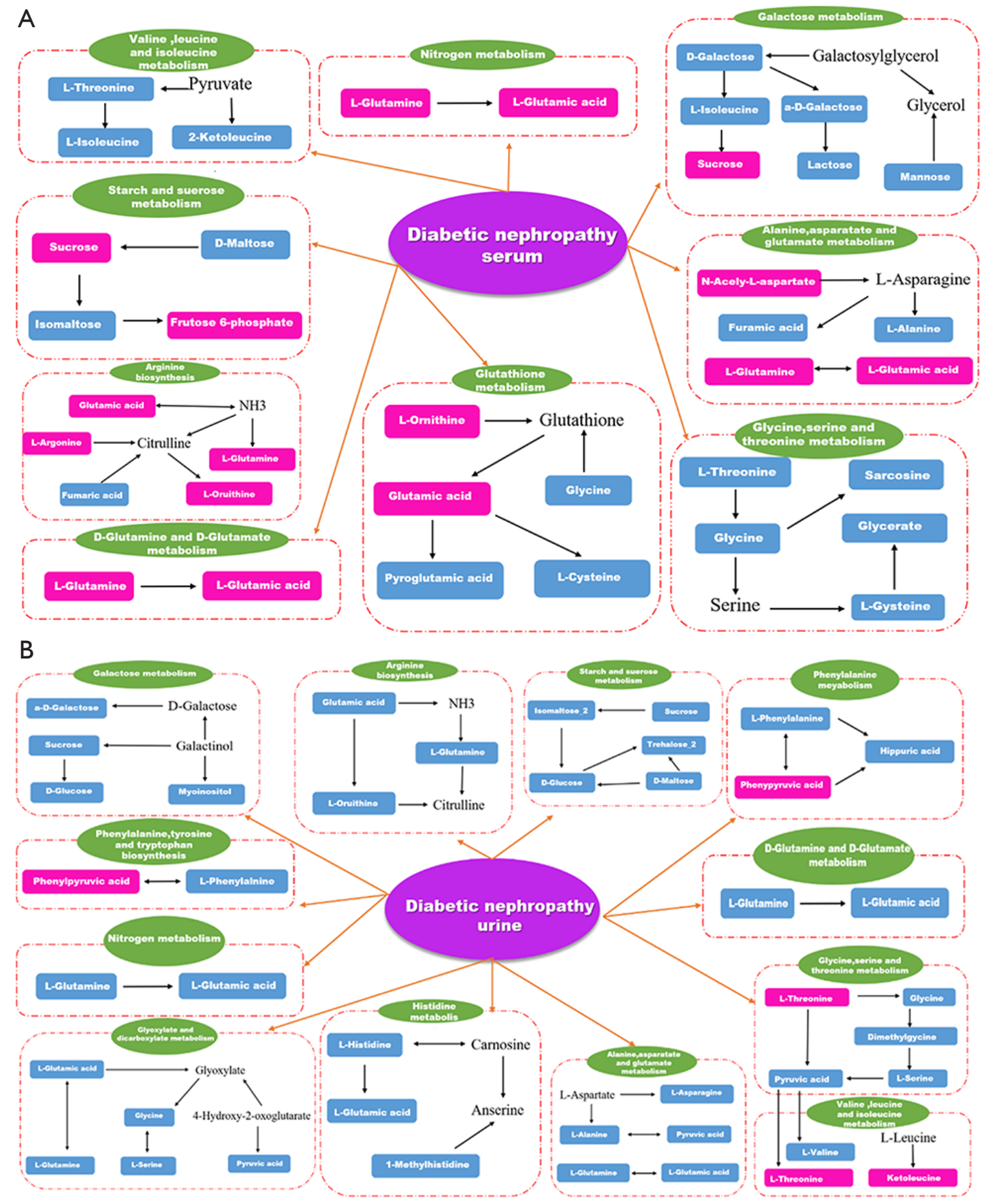

Figure 3 Serum and urinary top-ranked metabolic pathways. (A) For T2DM with nephropathy versus T2DM without nephropathy, the primary altered serum metabolism pathways are arginine biosynthesis; galactose metabolism; valine, leucine and isoleucine biosynthesis; starch and sucrose metabolism; alanine, aspartate and glutamate metabolism; glutathione metabolism; D-glutamine and D-glutamate metabolism; glycine, serine and threonine metabolism; and nitrogen metabolism $(\mathrm{P}<0.05)$. (B) For T2DM with nephropathy versus T2DM without nephropathy, the primary altered urinary metabolism pathways are starch and sucrose metabolism; valine, leucine and isoleucine biosynthesis; alanine, aspartate and glutamate metabolism; phenylalanine, tyrosine and tryptophan biosynthesis; glyoxylate and dicarboxylate metabolism; phenylalanine metabolism; glycine, serine and threonine metabolism; D-glutamine and D-glutamate metabolism; arginine biosynthesis; nitrogen metabolism; galactose metabolism; and histidine metabolism $(\mathrm{P}<0.05)$. Red indicates elevated metabolites, blue indicates reduced metabolites. 
A

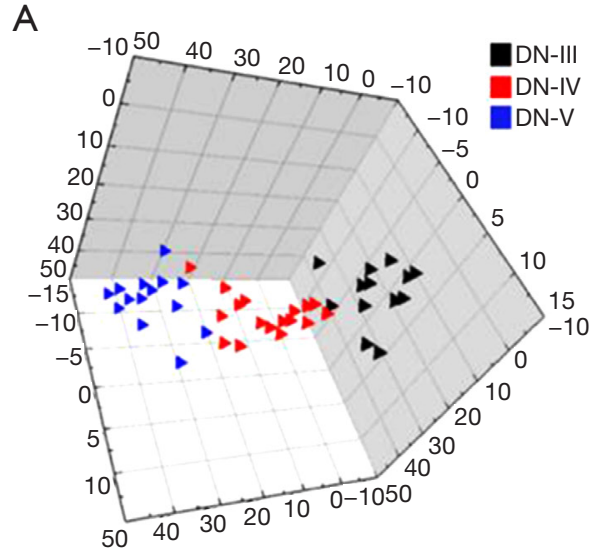

C

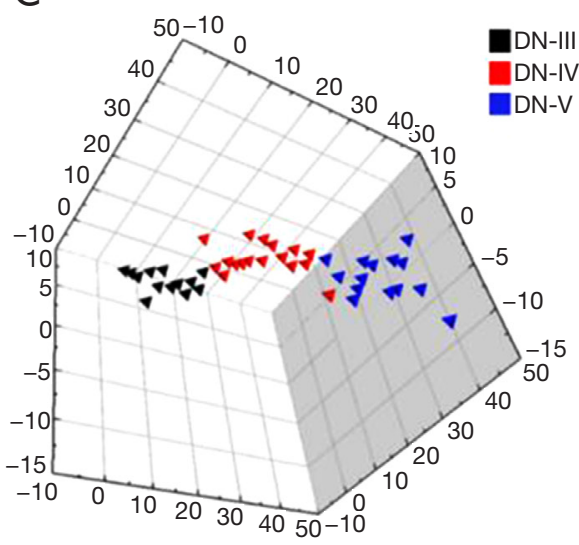

B
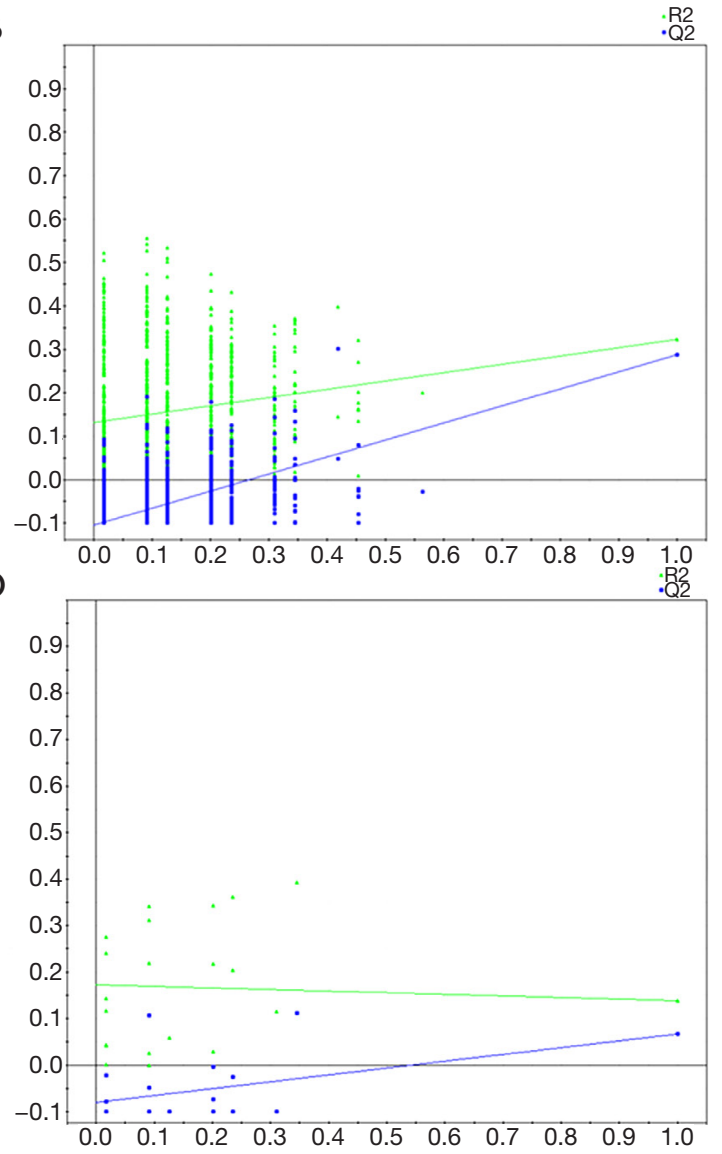

Figure 4 3D PLS-DA scores and validation plots for metabolic profiling of serum and urine in different stages of DN. (A) PLS-DA score plot of serum. (B) Validation plot of serum. (C) PLS-DA score plot of urine. (D) Validation plot of urine. Black triangles = DN-III, red triangles $=\mathrm{DN}-\mathrm{IV}$, blue triangles $=\mathrm{DN}-\mathrm{V}$.

characteristics of reducing proteinuria and glomerular disease in diabetes. In this study, GC-TOFMS-based metabolomics was used to detect DN-related metabolic changes in serum. Compared with T2DM patients, we observed a significantly decreased serum taurine levels in patients with $\mathrm{DN}$, which suggests that taurine metabolic disorders are associated with diabetic kidney damage. In addition, in order to further assess kidney damage in patients with $\mathrm{DN}$, we also compared the serum taurine levels in different stages of DN. The serum level of taurine decreased stepwise with the aggravation of kidney disease. This result also proves that taurine may be protective against $\mathrm{DN}$ (39).

We observed that the serum level of fumaric acid increased significantly in the pathways of arginine biosynthesis and alanine, aspartate, and glutamate metabolism of DN patients compared with the T2DM group. These findings indicate that disordered fumaric acid metabolism may be associated with diabetic renal impairment. Fumaric acid, a dicarboxylic acid, is a precursor to L-malate during the Krebs tricarboxylic acid (TCA) cycle. Zheng et al. (40) showed that the accumulated fumaric acid due to the inactivation of mutations enzyme fumarate hydratase $(\mathrm{FH})$ in the TCA cycle led to oxidative stress. You et al. (41) found that Nox4 inhibited the regulation of fumarate levels, indicating that the TCA cyclic enzyme FH was the downstream target of Nox4. These results indicate that continuous oxidative stress cause diabetic renal injury, while fumaric acid may serve as a possible biomarker for evaluating the progression of DN.

Benzoic acid is a preservative in foods widely and is detoxified by glycine conjugation in human body. After binding to glycine, benzoic acid is excreted as hippuric acid. In this study, serum benzoic acid was significantly elevated 
Table 5 Serum and urine metabolites that significantly changed among DN-III, DN-IV and DN-V groups

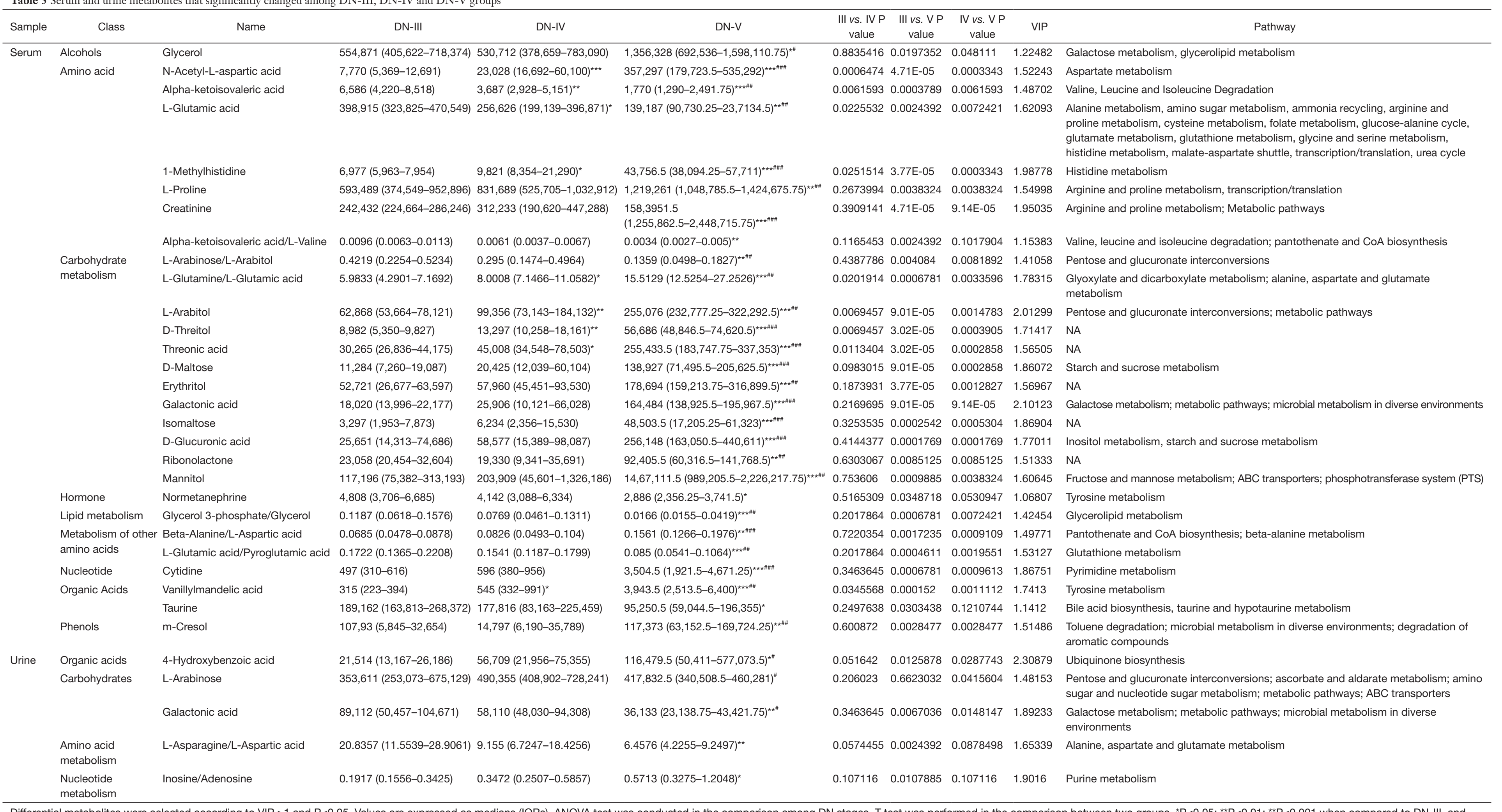

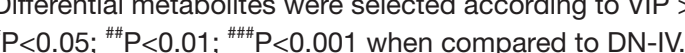


in DN patients, probably because of the consumption of serum glycine in DN patients, resulting in a decrease in the binding of benzoic acid to glycine, resulting in the accumulation of benzoic acid and causing renal toxicity $(42,43)$. Whether benzoic acid is a potential biomarker affecting $\mathrm{DN}$ progression requires further investigation.

Compared with T2DM patients without nephropathy, the levels of L-glutamic acid and pyroglutamic acid detected in serum samples from $\mathrm{DN}$ patients were significantly reduced. L-glutamic acid is a signaling molecule that regulates $\beta$-cells and secretes insulin to maintain blood sugar balance in the body (44). Insulin deficiency and increased gluconeogenesis through impaired cellular function can lead to increased L-glutamic acid catabolism, resulting in a significant decrease in L-glutamic acid contents (45). Our research found that serum L-glutamic acid levels differ in T2DM and DN patients, and from DN-III to DN-V, the serum L-glutamic acid content continues to decrease with the severity of kidney disease. Therefore, $\mathrm{L}$-glutamic acid is considered to be a potential biomarker for kidney injury in DN.

We also found changes in carbohydrates in the serum of DN patients, including fructose 6-phosphate, erythrose, and L-Arabitol. The abnormality of these carbohydrates suggested that saccharides plays a vital role in the process of DN. Fructose 6-phosphate, one of the class of organic compounds, is known as hexose phosphates, and is a carbohydrate derivative with a hexose replaced by one or more phosphate groups (46). Elbein (47) reported that glutamine-fructose-6-phosphate aminotransferase is the major rate-limiting enzyme of this pathway, which increases the expression of the glutamine-fructose-6-phosphate transaminase 1 (GFPT1) gene, thereby increasing the susceptibility to diabetes and diabetic nephropathy. We observed a significant decreased serum level of fructose 6-phosphate in DN patients compared with T2DM patients, which suggests that diabetic renal impairment may affect glucose-6-phosphate metabolism. Gluconic acid occurs naturally in fruit. Normally, glucose is provided by glycolysis and cyclic decomposition. In T2DM, there is an obstacle to the catabolism of glucose (48).

Glycerol 1-octadecanoate, a monoacylglyceride or a monoacylglycerol, is a type of glycerol that is covalently bonded to a glycerol molecule by an ester bond through a fatty acid chain (49). Monoacylglycerols are the main end-products in animals during the intestinal digestion of dietary fats by the enzyme pancreatic lipase. They are absorbed directly by intestinal cells and are transformed into triacylglycerols through the monoacylglycerol pathway before being transitted in lymph to the liver (50). Our data show that DN patients had lower serum levels of glycerol 1-octadecanoate than patients with T2DM without nephropathy, which suggests that glycerol 1-octadecanoate metabolism disorder may be related to diabetic kidney damage. Whether glycerol 1-octadecanoate can be a biomarker for the early diagnosis of DN needs further study.

In addition, metabolic disorders in the urine are also traceable. In the present study, T2DM patients with nephropathy excreted daily averages of more urinary 1-xylonate-2 and oxalic acid than those without nephropathy. Hyperoxaluria is a metabolic disorder with oxalate crystal deposition in various organs, including the kidney (51). The mechanism leading to hyperoxaluria is an increased load of free fatty acids in the intestine, resulting in reduced binding of calcium to free fatty acids and reduced synthesis of calcium oxalate. Then, an increasing amount of oxalic acid remains free and is absorbed in the intestine, leading to hyperoxaluria (52). Therefore, inhibition of oxalic acid may be a promising treatment strategy for patients with DN.

The levels of L-valine, L-histidine, glycine, L-asparagine, and L-aspartic acid found in urine samples from DN patients were observably elevated, indicating that urine amino acid metabolism associated with the development of DN.

The kidney is an important site of amino acid metabolism. Therefore, abnormal amino acid metabolism may be predicted as kidney damage. L-glycine has been shown the protection roles against several complications of diabetes in recent years $(53,54)$. L-glycine protects the kidneys, but the precise mechanisms by which L-glycine ameliorates DN remain unclear (45). In our population, we observed a significant increase in urine L-glycine concentration in DN patients compared with T2DM patients. Therefore, L-glycine can not only be a drug for the treatment of diabetic complications but also a potential diagnostic biomarker for $\mathrm{DN}$.

The levels of urine glucose, such as D-glucose, sucrose and gluconic acid, found in urine samples of patients with DN were significantly increased. Wei et al. (55) showed that increasing urine levels of TCA cycle intermediates in DN mice, including citrate, cis-aconitate, and fumarate, which might reflect the system pressure caused by hyperglycemia or local influences on kidney impaired tubule transport and mitochondrial function (56). In the ESRD group, the urine contents of galactonic acid and allose were significantly lower than those in the early $\mathrm{DN}$ and clinical $\mathrm{DN}$ groups. Thus, urine saccharides can be recognized as potential biomarkers for evaluating the progress of $\mathrm{DN}$ (57-59). 
There are still several limitations in our study. First, the number of patients was relatively small in each group. Second, although we found that serum taurine concentrations were lower in DN patients than in those without nephropathy and decreased along with the progress of nephropathy, direct evidence is lacking to support the association between serum taurine concentration and deterioration of renal function. Third, the study lacks a robust validation in an independent population. In the near future, we plan to select several metabolites that have increased or decreased the most from the GC-TOFMS metabolomics analysis and use targeted metabolomics to quantitatively evaluate the serum or urine levels of those metabolites. In addition, we will combine molecular biology approaches and other methods to further explore factors and the relevant molecular mechanisms that influence the changes of these metabolites and to explain the reasons for the changes of these metabolites.

In addition, we also collected clinical serum and urine samples in other diabetic complications, such as patients with diabetic peripheral neuropathy, and used methods similar to GC-TOFMS-based metabolomics to reveal the metabolic differences between T2DM patients with or without peripheral neuropathy. Some interesting and novel results were found between the two groups, which we will show in the near future.

\section{Conclusions}

In the present study, we integrated information regarding serum and urine metabolites using GC-TOFMS-based metabolomics to reveal the differences between T2DM patients with or without nephropathy and metabolic changes in the development of DN. Our results showed that several metabolic disorders occur, including altered organic acid metabolism and disordered glucose metabolism, during the onset and pathogenesis of DN. These results show that GC-TOFMS-based metabolomics is a promising way to reveal potential metabolic pathways to assess the risk of DN complications and to evaluate therapeutic effects.

\section{Acknowledgments}

Funding: This work was supported by the National Natural Science Foundation of China (81620108030, 81873076), the Key project of Shanghai 3-year plan (ZY2018-2020CCCX-2002-01) and Shanghai Talents Development Fund Project (2017090).

\section{Footnote}

Conflicts of Interest: The authors have no conflicts of interest to declare.

Ethical Statement: The authors are accountable for all aspects of the work in ensuring that questions related to the accuracy or integrity of any part of the work are appropriately investigated and resolved. All patients who participated in the study offered a signed informed consent form. The ethical approval for the present study was provided by the Ethics Committee of Shanghai University of Traditional Chinese Medicine.

Open Access Statement: This is an Open Access article distributed in accordance with the Creative Commons Attribution-NonCommercial-NoDerivs 4.0 International License (CC BY-NC-ND 4.0), which permits the noncommercial replication and distribution of the article with the strict proviso that no changes or edits are made and the original work is properly cited (including links to both the formal publication through the relevant DOI and the license). See: https://creativecommons.org/licenses/by-nc-nd/4.0/.

\section{References}

1. International Diabetes Federation. IDF Diabetes Atlas Eighth Edition. 2017.

2. Rathmann W, Giani G. Global prevalence of diabetes: estimates for the year 2000 and projections for 2030. Diabetes Care 2004;27:2568-9; author reply 2569.

3. Thomas MC, Brownlee M, Susztak K, et al. Diabetic kidney disease. Nat Rev Dis Primers 2015;1:15018.

4. American Diabetes A. 10. Microvascular Complications and Foot Care: Standards of Medical Care in Diabetes-2018. Diabetes Care 2018;41:S105-18.

5. Bakris GL. Recognition, pathogenesis, and treatment of different stages of nephropathy in patients with type 2 diabetes mellitus. Mayo Clin Proc 2011;86:444-56.

6. Zhang L, Wang H, Long J, et al. China Kidney Disease Network(CK-NET) 2014 Annual Data Report. Am J Kidney Dis 2017;69:A4.

7. Foundation NK. KDOQI Clinical Practice Guideline for Diabetes and CKD: 2012 update. Am J Kidney Dis 2012;60:850-86.

8. Altemtam N, Russell J, El Nahas M. A study of the natural history of diabetic kidney disease(DKD). Nephrol Dial Transplant 2012;27:1847-54. 
9. Chou CA, Lin CN, Chiu DT, et al. Tryptophan as a surrogate prognostic marker for diabetic nephropathy. J Diabetes Investig 2018;9:366-74.

10. Liu J, Wang D, Chen Y, et al. 1H NMR-based metabonomic analysis of serum and urine in a nonhuman primate model of diabetic nephropathy. Mol Biosyst 2013;9:2645-52.

11. Fan Y, Li Y, Chen Y, et al. Comprehensive Metabolomic Characterization of Coronary Artery Diseases. J Am Coll Cardiol 2016;68:1281-93.

12. Tam ZY, Ng SP, Tan LQ, et al. Metabolite profiling in identifying metabolic biomarkers in older people with lateonset type 2 diabetes mellitus. Sci Rep 2017;7:4392.

13. Rossi C, Marzano V, Consalvo A, et al. Proteomic and metabolomic characterization of streptozotocin-induced diabetic nephropathy in TIMP3-deficient mice. Acta Diabetol 2018;55:121-9.

14. Li L, Wang C, Yang H, et al. Metabolomics reveal mitochondrial and fatty acid metabolism disorders that contribute to the development of DKD in T2DM patients. Mol Biosyst 2017;13:2392-400.

15. Wu T, Xie G, Ni Y, et al. Serum metabolite signatures of type 2 diabetes mellitus complications. J Proteome Res 2015;14:447-56.

16. Wu T, Qiao S, Shi C, et al. Metabolomics window into diabetic complications. J Diabetes Investig 2018;9:244-55.

17. Xu Y, Wang L, He J, et al. Prevalence and control of diabetes in Chinese adults. JAMA 2013;310:948-59.

18. de Boer IH, Rue TC, Hall YN, et al. Temporal Trends in the Prevalence of Diabetic Kidney Disease in the United States. JAMA 2011;305:2532-9.

19. Sinn DH, Kang D, Jang HR, et al. Development of chronic kidney disease in patients with non-alcoholic fatty liver disease: A cohort study. J Hepatol 2017;67:1274-80.

20. Huang GM, Huang KY, Lee TY, et al. An interpretable rule-based diagnostic classification of diabetic nephropathy among type 2 diabetes patients. BMC Bioinformatics 2015;16 Suppl 1:S5.

21. He F, Xia X, Wu XF, et al. Diabetic retinopathy in predicting diabetic nephropathy in patients with type 2 diabetes and renal disease: a meta-analysis. Diabetologia 2013;56:457-66.

22. Chawla V, Roshan B. Non-proteinuric diabetic nephropathy. Curr Diab Rep 2014;14:529.

23. Wu Q, Zhang H, Dong X, et al. UPLC-Q-TOF/MS based metabolomic profiling of serum and urine of hyperlipidemic rats induced by high fat diet. J Pharm Anal 2014;4:360-7.
24. Wang X, Lv H, Zhang A, et al. Metabolite profiling and pathway analysis of acute hepatitis rats by UPLC-ESI MS combined with pattern recognition methods. Liver Int 2014;34:759-70.

25. Luan H, Liu LF, Tang Z, et al. Comprehensive urinary metabolomic profiling and identification of potential noninvasive marker for idiopathic Parkinson's disease. Sci Rep 2015;5:13888.

26. Xia J, Wishart DS. Using MetaboAnalyst 3.0 for Comprehensive Metabolomics Data Analysis. Curr Protoc Bioinformatics 2016;55:14.10.1-14.10.91.

27. Dhama K, Sachan S, Khandia R, et al. Medicinal and Beneficial Health Applications of Tinospora cordifolia(Guduchi): A Miraculous Herb Countering Various Diseases/Disorders and its Immunomodulatory Effects. Recent Pat Endocr Metab Immune Drug Discov 2017;10:96-111.

28. Chen S, Hong SW, Iglesias-de la Cruz MC, et al. The key role of the transforming growth factor-beta system in the pathogenesis of diabetic nephropathy. Ren Fail 2001;23:471-81.

29. Pundir CS, Jakhar S, Narwal V. Determination of urea with special emphasis on biosensors: A review. Biosens Bioelectron 2019;123:36-50.

30. Razi F, Nasli-Esfahani E, Bandarian F. Association of serum uric acid with nephropathy in Iranian type 2 diabetic patients. J Diabetes Metab Disord 2018;17:71-5.

31. Sueud T, Hadi NR, Abdulameer R, et al. Assessing urinary levels of IL-18, NGAL and albumin creatinine ratio in patients with diabetic nephropathy. Diabetes Metab Syndr 2019;13:564-8.

32. Cetin E, Civelek S, Andican G, et al. Plasma AGEpeptides and C-peptide in early-stage diabetic nephropathy patients on thiamine and pyridoxine therapy. Minerva Med 2013;104:93-101.

33. Hirayama A, Nakashima E, Sugimoto $M$, et al. Metabolic profiling reveals new serum biomarkers for differentiating diabetic nephropathy. Anal Bioanal Chem 2012;404:3101-9.

34. Gong P, Chang X, Chen X, et al. Metabolomics study of cadmium-induced diabetic nephropathy and protective effect of caffeic acid phenethyl ester using UPLC-QTOF-MS combined with pattern recognition. Environ Toxicol Pharmacol 2017;54:80-92.

35. Koh JH, Lee ES, Hyun M, et al. Taurine alleviates the progression of diabetic nephropathy in type 2 diabetic rat model. Int J Endocrinol 2014;2014:397307.

36. Wu G, Yang J, Sun C, et al. Effect of taurine on alcoholic 
liver disease in rats. Adv Exp Med Biol 2009;643:313-22.

37. Lin S, Yang J, Wu G, et al. Preventive effect of taurine on experimental type II diabetic nephropathy. J Biomed Sci 2010;17 Suppl 1:S46.

38. Winiarska K, Szymanski K, Gorniak P, et al. Hypoglycaemic, antioxidative and nephroprotective effects of taurine in alloxan diabetic rabbits. Biochimie 2009;91:261-70.

39. Han X, Ito T, Azuma J, et al. The quest for an animal model of diabetic nephropathy and the role of taurine deficiency. Adv Exp Med Biol 2015;803:217-26.

40. Zheng L, Cardaci S, Jerby L, et al. Fumarate induces redox-dependent senescence by modifying glutathione metabolism. Nat Commun 2015;6:6001.

41. You YH, Quach T, Saito R, et al. Metabolomics Reveals a Key Role for Fumarate in Mediating the Effects of NADPH Oxidase 4 in Diabetic Kidney Disease. J Am Soc Nephrol 2016;27:466-81.

42. Mitch WE, Brusilow S. Benzoate-induced changes in glycine and urea metabolism in patients with chronic renal failure. J Pharmacol Exp Ther 1982;222:572-5.

43. Irwin C, van Reenen M, Mason S, et al. Contribution towards a Metabolite Profile of the Detoxification of Benzoic Acid through Glycine Conjugation: An Intervention Study. PLoS One 2016;11:e167309.

44. Rhee SY, Jung ES, Park HM, et al. Plasma glutamine and glutamic acid are potential biomarkers for predicting diabetic retinopathy. Metabolomics 2018;14:89.

45. Du Y, Xu BJ, Deng X, et al. Predictive metabolic signatures for the occurrence and development of diabetic nephropathy and the intervention of Ginkgo biloba leaves extract based on gas or liquid chromatography with mass spectrometry. J Pharm Biomed Anal 2019;166:30-9.

46. Tsuruoka M, Hara J, Hirayama A, et al. Capillary electrophoresis-mass spectrometry-based metabolome analysis of serum and saliva from neurodegenerative dementia patients. Electrophoresis 2013;34:2865-72.

47. Elbein SC, Zheng H, Jia Y, et al. Molecular screening of the human glutamine-fructose-6-phosphate amidotransferase 1(GFPT1) gene and association studies with diabetes and diabetic nephropathy. Mol Genet Metab 2004;82:321-8.

48. Mallipattu SK, Gallagher EJ, LeRoith D, et al. Diabetic nephropathy in a nonobese mouse model of type 2 diabetes mellitus. Am J Physiol Renal Physiol 2014;306:F1008-17.
49. Sinha R, Ahn J, Sampson JN, et al. Fecal Microbiota, Fecal Metabolome, and Colorectal Cancer Interrelations. PLoS One 2016;11:e0152126.

50. Wang X, Wang J, Rao B, et al. Gut flora profiling and fecal metabolite composition of colorectal cancer patients and healthy individuals. Exp Ther Med 2017;13:2848-54.

51. Albersmeyer M, Hilge R, Schrottle A, et al. Acute kidney injury after ingestion of rhubarb: secondary oxalate nephropathy in a patient with type 1 diabetes. BMC Nephrol 2012;13:141.

52. Muji A, Moll S, Saudan P. (Oxalate nephropathy: a new entity of acute kidney injury in diabetic patients?). Rev Med Suisse 2015;11:493-4, 6-8.

53. Pérez-Torres I, Ibarra B, Soria-Castro E, et al. Effect of glycine on the cyclooxygenase pathway of the kidney arachidonic acid metabolism in a rat model of metabolic syndrome. Can J Physiol Pharmacol 2011;89:899-910.

54. Wang Z, Zhang J, Wang L, et al. Glycine mitigates renal oxidative stress by suppressing Nox 4 expression in rats with streptozotocin-induced diabetes. J Pharmacol Sci 2018;137:387-94.

55. Wei T, Zhao L, Jia J, et al. Metabonomic analysis of potential biomarkers and drug targets involved in diabetic nephropathy mice. Sci Rep 2015;5:11998.

56. Salek RM, Maguire ML, Bentley E, et al. A metabolomic comparison of urinary changes in type 2 diabetes in mouse, rat, and human. Physiol Genomics 2007;29:99-108.

57. Rainesalo S, Keranen T, Palmio J, et al. Plasma and cerebrospinal fluid amino acids in epileptic patients. Neurochem Res 2004;29:319-24.

58. Lim SR, Hyun SH, Lee SG, et al. Potential urinary biomarkers of nephrotoxicity in cyclophosphamide-treated rats investigated by NMR-based metabolic profiling. J Biochem Mol Toxicol 2017. doi: 10.1002/jbt.21871.

59. Watanabe M, Suliman ME, Qureshi AR, et al. Consequences of low plasma histidine in chronic kidney disease patients: associations with inflammation, oxidative stress, and mortality. Am J Clin Nutr 2008;87:1860-6.

Cite this article as: Shao M, Lu H, Yang M, Liu Y, Yin P, Li G, Wang Y, Chen L, Chen Q, Zhao C, Lu Q, Wu T, Ji G. Serum and urine metabolomics reveal potential biomarkers of T2DM patients with nephropathy. Ann Transl Med 2020;8(5):199. doi: 10.21037/atm.2020.01.42 
Table S1 Clinical information of T2DM patients with and without nephropathy and among DN-III, DN-IV and DN-V groups

\begin{tabular}{|c|c|c|c|c|c|c|c|c|c|}
\hline \multirow{2}{*}{ Characteristics } & \multirow{2}{*}{$\begin{array}{l}\text { T2DM without nephropathy } \\
\qquad(\mathrm{n}=44)\end{array}$} & \multicolumn{4}{|c|}{ T2DM with nephropathy $(\mathrm{n}=44)$} & \multicolumn{4}{|c|}{$P$ value } \\
\hline & & Overall & DN-III & DN-IV & DN-V & DN vs. T2DM & III vs. IV & III vs. V & IV vs. V \\
\hline Age (years) & $71(55.75-79.5)$ & $62(55.75-71.25)$ & 71 [56-81] & $62[58-70]$ & $61(55.25-67)$ & 0.176 & 0.519 & 0.519 & 0.72 \\
\hline Glucose (mmol/L) & $7.45(4.95-9.7)$ & $7.8(5.475-9.525)$ & $8.2(6.7-11.9)$ & $7.5(4.9-9.6)$ & $7.25(4.95-8.775)$ & 0.826 & 0.232 & 0.185 & 0.487 \\
\hline 2hPG (mmol/L) & $11(7.125-15.175)$ & $14(10.45-16.875)$ & $15.3(13.3-16.35)$ & $16.1(13.3-19.8)$ & $9.05(6.575-12.95)^{* * \# \#}$ & 0.06 & 0.716 & 0.008 & 0.008 \\
\hline C-peptide (pmol/mL) & $1.39(0.85-1.98)$ & $1.63(0.89-2.54)$ & $1.195(0.79-1.57)$ & $1.575(0.8225-2.405)$ & $3.44(1.95-4.46)^{\star * *}$ & 0.17 & 0.202 & 0.004 & 0.021 \\
\hline $\mathrm{HbAIC}$ (mg/dL, \%) & $8.3(7.05-9.5)$ & $7.8(6.6-9.7)$ & $9.9(9.1-11.4)$ & $7.7(7-9.375)^{\star}$ & $6.65(6.15-7.625)^{* * *}$ & 0.618 & 0.043 & 0.005 & 0.043 \\
\hline WBC $\left({ }^{*} 10^{9} / L\right)$ & $7.27(6.212-8.748)$ & $6.1(5.1-8)$ & $5.9(5.6-7.4)$ & $6.1(5.1-8)$ & $7.25(4.725-8.4)$ & 0.065 & 0.802 & 0.802 & 0.802 \\
\hline $\mathrm{Neu}(\%)$ & $63.1(58.025-71.35)$ & 65 (58.6-73.75) & $62.3(53.6-74.9)$ & $67.6(57.2-70.4)$ & $64.4(62.4-79.2)$ & 0.714 & 0.967 & 0.522 & 0.622 \\
\hline Lym (\%) & $23.05(17.5-29.4)$ & $23.1(16.1-31.5)$ & $29.5(15.8-38.9)$ & $23.3(20.5-32.3)$ & $19.4(14.875-24)$ & 0.89 & 0.544 & 0.168 & 0.168 \\
\hline Mon (\%) & $8(7.175-9.85)$ & $6.5(5.5-8.025)$ & $5.7(5.3-7)$ & $6(5.5-7.7)$ & $7.95(6.05-9.1)$ & 0.001 & 0.721 & 0.081 & 0.143 \\
\hline HCT (\%) & $34.25(31.025-36.3)$ & $32.1(27.7-38.65)$ & $36.7(30-42.2)$ & $32(26.7-38.8)$ & $30.15(27.7-35.75)$ & 0.751 & 0.19 & 0.132 & 0.62 \\
\hline MCV (\%) & $86.35(82.725-89.75)$ & $87.8(85.2-90.575)$ & $88.1(85.2-89.8)$ & $86.1(84.4-89.2)$ & $90.75(86.75-93.65)$ & 0.131 & 0.295 & 0.198 & 0.052 \\
\hline HGB-Mass (pg) & $29(27.8-30.45)$ & $29.7(28.8-31)$ & $30.5(29.8-31.8)$ & $29.6(28.8-31)$ & $29.2(28.65-29.9)^{\star}$ & 0.042 & 0.147 & 0.023 & 0.35 \\
\hline HGB-Conc (g/L) & $335(328.5-344.25)$ & $336.5(321-351.25)$ & 351 [347-358] & 340 [329-354] & $322[318-333]^{\star \star *}$ & 0.655 & 0.116 & 0.002 & 0.034 \\
\hline RDW-CV (\%) & $13.55(12.85-14.525)$ & $13.05(12.575-13.9)$ & $12.5(12.3-12.9)$ & $13(12.6-13.8)$ & $13.7(13.4-14.5)^{\star \star \#}$ & 0.306 & 0.126 & 0.003 & 0.037 \\
\hline $\operatorname{PLT}\left({ }^{*} 10^{9} / \mathrm{L}\right)$ & $246(194.5-302.5)$ & $193(153.5-224)$ & $184[154-197]$ & 199 [157-233] & $194(152.5-258.75)$ & 0.003 & 0.497 & 0.497 & 0.889 \\
\hline MPV (fL) & $10.5(9.7-11.125)$ & $11.1(10.2-12.15)$ & $12.3(11.8-13.1)$ & $10.5(9.8-11.4)^{*}$ & $10.9(10.125-11.9)^{*}$ & 0.01 & 0.011 & 0.019 & 0.462 \\
\hline hs-CRP (mg/L) & $7(4-34.5)$ & $2(1-9.5)$ & $1.5(1-6.25)$ & $1[1-5]$ & $5[1-17]$ & $<0.001$ & 0.556 & 0.556 & 0.451 \\
\hline TP (g/L) & $65.55(60.5-68.825)$ & 64 [56-69] & $66.5(63.75-70)$ & $65[55-72]$ & $58(53.5-64.75)^{*}$ & 0.364 & 0.412 & 0.017 & 0.398 \\
\hline ALB (g/L) & $35.7(32.25-37.225)$ & 35 (29.5-38) & $38(35.75-39.5)$ & $37[30-40]$ & $30.5(28.25-33.75)^{\star \star \star}$ & 0.69 & 0.258 & 0.001 & 0.142 \\
\hline GLB (g/L) & $30.2(27.35-34.825)$ & $29(25-31.5)$ & $29(27-30.5)$ & 28 [25-31] & $28(24-31.5)$ & 0.047 & 0.811 & 0.811 & 0.811 \\
\hline $\mathrm{DBIL}(\mu \mathrm{mol} / \mathrm{L})$ & $3.1(2.1-3.8)$ & $1.6(1.2-2)$ & $2.1(1.45-2.75)$ & $1.7(1.5-1.9)$ & $1.2(0.925-1.45)^{\star * \#}$ & $<0.001$ & 0.351 & 0.017 & 0.019 \\
\hline TBA $(\mu \mathrm{mol} / \mathrm{L})$ & $3.15(2.475-3.925)$ & $4(2.5-7)$ & $6(6-9.25)$ & $4[2-8]$ & $3(2-3.75)^{\star \star}$ & 0.244 & 0.066 & 0.002 & 0.132 \\
\hline $\mathrm{Cr}(\mu \mathrm{mol} / \mathrm{L})$ & 72 (58.5-94.25) & $119(69-431.75)$ & $66[58-88]$ & 107 [70-243] & $714.5(431.75-854)^{\star \star \star * \# \# ~}$ & 0.002 & 0.063 & $<0.001$ & $<0.001$ \\
\hline Urea (mmol/L) & $6.85(4.525-8.15)$ & $9.15(6.275-20.65)$ & $6.4(5.1-6.8)$ & $9.1(8-12.8)^{\star}$ & $21.45(16.725-24.05)^{\star \star * * \# \# \#}$ & 0.001 & 0.013 & $<0.001$ & $<0.001$ \\
\hline UA ( $(\mu \mathrm{mol} / \mathrm{L})$ & $262.5(210.75-327.75)$ & $382(309-432.25)$ & 311 [284-403] & 380 [330-425] & $437.5[384-459]^{\star}$ & $<0.001$ & 0.108 & 0.013 & 0.153 \\
\hline $\mathrm{Ca}(\mathrm{mmol} / \mathrm{L})$ & $2.24(2.16-2.395)$ & $2.165(2.087-2.273)$ & $2.165(2.095-2.278)$ & $2.165(2.04-2.313)$ & $2.16(2.09-2.188)$ & 0.02 & 0.921 & 0.921 & 0.921 \\
\hline $\mathrm{P}(\mathrm{mmol} / \mathrm{L})$ & $1.22(1.1-1.3725)$ & $1.225(1.07-1.565)$ & $1.08(1.04-1.108)$ & $1.265(1.135-1.41)$ & $1.54(1.19-1.875)$ & 0.65 & 0.111 & 0.105 & 0.111 \\
\hline $\mathrm{K}(\mathrm{mmol} / \mathrm{L})$ & $4(3.75-4.2)$ & $4.2(3.8-4.45)$ & $4.3(4.025-4.325)$ & $4.1(3.9-4.5)$ & $4.1(3.575-4.975)$ & 0.223 & 0.979 & 0.979 & 0.979 \\
\hline $\mathrm{Na}(\mathrm{mmol} / \mathrm{L})$ & $139(137-140)$ & $141(138.5-142)$ & $140(137.5-141)$ & $142[140-142]$ & $140(137.5-141.75)$ & 0.028 & 0.26 & 0.938 & 0.403 \\
\hline $\mathrm{Cl}(\mathrm{mmol} / \mathrm{L})$ & $102(100.5-104)$ & $104(101-104.5)$ & $103(101-104.25)$ & 104 [101-107] & $103.5(97.25-104)$ & 0.443 & 0.461 & 0.461 & 0.322 \\
\hline $\mathrm{TG}(\mathrm{mmol} / \mathrm{L})$ & $1.03(0.847-1.378)$ & $1.61(1.305-2.41)$ & $1.945(1.235-2.503)$ & $2.11(1.49-2.42)$ & $1.355(1.267-1.663)$ & $<0.001$ & 0.565 & 0.565 & 0.129 \\
\hline $\mathrm{TC}(\mathrm{mmol} / \mathrm{L})$ & $3.945(3.522-4.873)$ & $4.95(3.83-5.57)$ & $5.225(3.917-5.645)$ & $5.01(4.43-5.76)$ & $4.38(3.607-5.198)$ & 0.019 & 0.965 & 0.473 & 0.473 \\
\hline $\mathrm{HDL}(\mathrm{mmol} / \mathrm{L})$ & $1(0.84-1.14)$ & $1.05(0.925-1.215)$ & $1.13(1.012-1.333)$ & $1.11(0.85-1.35)$ & $1(0.95-1.073)$ & 0.573 & 0.690 & 0.227 & 0.425 \\
\hline LDL (mmol/L) & $2.545(2.015-3.26)$ & $3.15(2.42-3.74)$ & $3.55(2.5075-3.84)$ & $3.07(2.55-3.71)$ & $3(2.12-3.425)$ & 0.033 & 0.626 & 0.626 & 0.626 \\
\hline ApoA (g/L) & $1.06(0.885-1.253)$ & $1.12(1.01-1.223)$ & $1.17(1.12-1.393)$ & $1.12(1.002-1.313)$ & $1.04(0.99-1.15)$ & 0.59 & 0.544 & 0.284 & 0.353 \\
\hline ApoB (g/L) & $0.785(0.635-0.873)$ & $0.95(0.747-1.093)$ & $0.95(0.76-1.13)$ & $0.97(0.852-1.108)$ & $0.94(0.682-1.048)$ & 0.01 & 0.66 & 0.66 & 0.66 \\
\hline Lp-A (mmol/L) & $269(111-415.25)$ & $149.5(35.75-364)$ & $69(35.25-151.75)$ & $173(30.5-364)$ & $200.5(50-386.25)$ & 0.071 & 0.589 & 0.589 & 0.589 \\
\hline eGFR (mL/min/1.73 $\left.\mathrm{m}^{2}\right)$ & $8.775(6.627-10.693)$ & $6.405(2.93-8.71)$ & $7.91(6.17-10.02)$ & $4.72(2.55-8.57)$ & $6.46(1.887-8.593)$ & 0.028 & 0.054 & 0.285 & 0.451 \\
\hline
\end{tabular}

Data were expressed as the medians (IQRs) at normal distribution. The Wilcoxon test was used to compare these variables between T2DM patients with and without nephropathy, and P<0.05 was considered

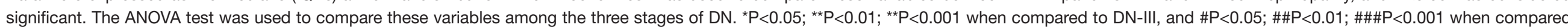
to DN-IV. M, male; F, female; BMI, body mass index; 2hPG, two hours post-load plasma glucose; HbAlC, glycosylated hemoglobin or glycated hemoglobin; WBC, white blood cell; Neu, neutrophil ratio; Lym, Iymphocyte ratio; Mon, monocyte ratio; Eso, eosinophil ratio; Bas, basophil ratio; RBC, red blood cell; HGB, hemoglobin; HCT, hematocrit; MCV, mean corpuscular volume; HGB-Mass, RBC average HGB lymphocyte ratio; Mon, monocyte ratio; Eso, eosinophil ratio; Bas, basophil ratio; RBC, red blood cell; HGB, hemoglobin; HCT, hematocrit; MCV, mean corpuscular volume; HGB-Mass, RBC average HGB
content; HGB-Conc, RBC average HGB concentration; RDW-CV, red blood cell distribution width coefficient of variation; PLT, platelet; MPV, mean platelet volume; hs-CRP, hypersensitive C-reactive protein; TP, total protein; ALB, albumin; GLB, globulin; A-G, albumin/globulin; AST, aspartate aminotransferase; ALT, alanine aminotransferase; AST-ALT, aspartate aminotransferase/alanine aminotransferase; $r-G T$, Glutamyl transpeptidase; AKP, alkaline phosphatase; TBIL, total bilirubin; DBIL, direct bilirubin; TBA, total bile acid; Cr, creatinine; UA, uric acid; TG, triglyceride; TC, total cholesterol; HDL, high-density lipoprotein; LDL, low-density lipoprotein; ApoA, apolipoprotein a; ApoB, apolipoprotein b; Lp-A, lipoprotein a; eGFR, estimated glomerular filtration rate.
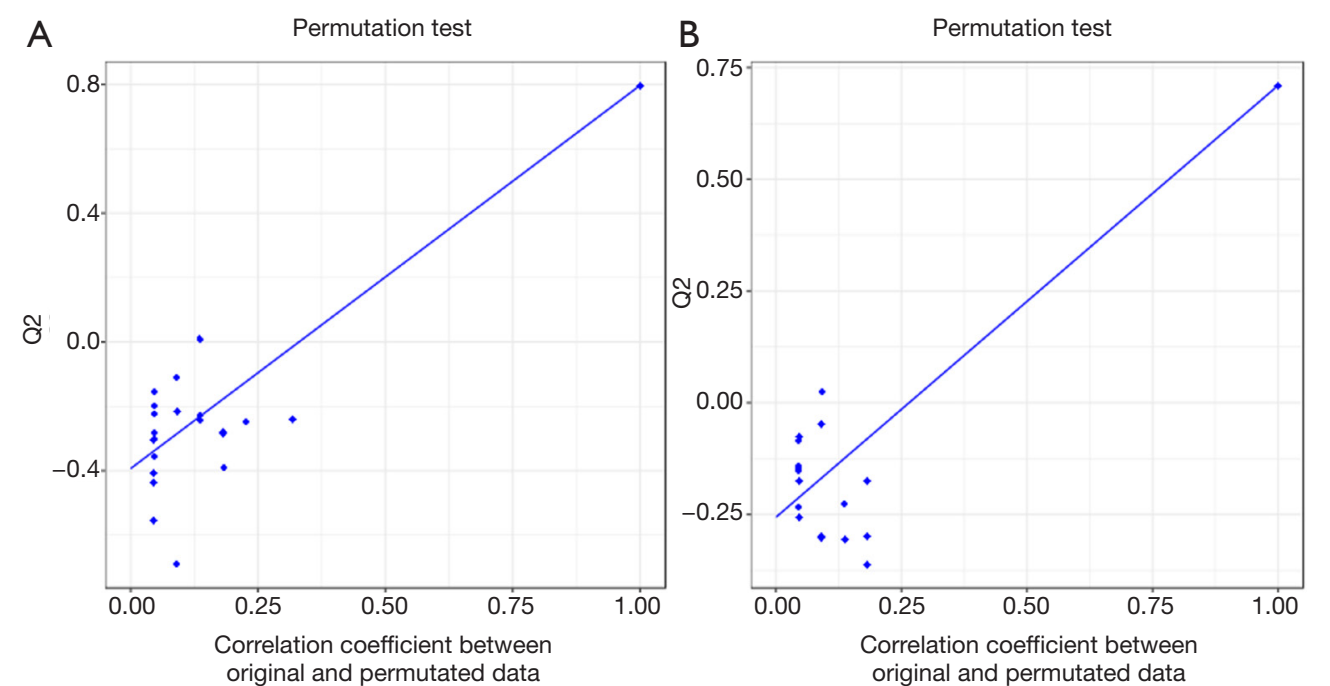

Figure S1 The permutation testing (20 times) result of the OPLS-DA model. (A) The permutation testing result of the serum OPLS-DA

model. (B) The permutation testing result of the urine OPLS-DA model. 
Table S2 Differential serum metabolites betweenT2DM with and without nephropathy

\begin{tabular}{|c|c|c|c|c|c|c|c|c|c|}
\hline Name & Class & T test. $P$ & VIP & FC & DN & T2DM & HMDBID & Kegg ID & Pathway \\
\hline 1-Methylhistidine & Amino acid & $1.21 \mathrm{E}-04$ & 1.3 & 0.6 & $15,612(7,125.5,39,741)$ & $8,910.5(6,143,12,853.75)$ & HMDB00001 & $\mathrm{C} 01152$ & Histidine metabolism \\
\hline Alpha-ketoisovaleric acid & Amino acid & $1.00 \mathrm{E}-03$ & 1.4 & 0.6 & $3,640(1,992.5,6,487)$ & $2,190.5(1,425.25,3,217.5)$ & HMDB00019 & C00141 & Valine, leucine and isoleucine degradation \\
\hline Alpha-ketoisovaleric acid/L-Valine & $\begin{array}{l}\text { Amino acid metabolism; metabolism of } \\
\text { cofactors and vitamins }\end{array}$ & $4.08 \mathrm{E}-04$ & 1.3 & 0.6 & $0.01(0.003,0.01)$ & $0(0.002,0.005)$ & HMDB00019/HMDB00883 & C00141/C00183 & Valine, leucine and isoleucine degradation; pantothenate and CoA biosynthesis \\
\hline Beta-Alanine & Amino acid & $1.50 \mathrm{E}-04$ & 1.4 & 0.7 & $10,038(8,363.25,15,552.5)$ & $7,151.5(6,114,8,661)$ & HMDB00056 & C00099 & Aspartate metabolism, beta-alanine metabolism, propanoate metabolism, pyrimidine metabolism \\
\hline Beta-Alanine/L-Aspartic acid & $\begin{array}{l}\text { Metabolism of cofactors and vitamins; } \\
\text { Metabolism of other amino acids }\end{array}$ & $4.86 \mathrm{E}-05$ & 1.5 & 0.5 & $0.09(0.061,0.144)$ & $0.05(0.037,0.076)$ & HMDB00056/HMDB00191 & C00099/C00049 & Pantothenate and COA biosynthesis; beta-alanine metabolism \\
\hline Cytidine & Nucleotide & $5.05 \mathrm{E}-04$ & 1.2 & 0.7 & $761.5(400.75,2396.25)$ & $498(398,615.5)$ & HMDB00089 & C00475 & Pyrimidine metabolism \\
\hline Fructose 6-phosphate & Carbohydrates & $2.50 \mathrm{E}-10$ & 2 & 1.8 & $516,665.5(371,837.5,627,208)$ & $934,897(717,232,1,136,517.25)$ & HMDB00124 & C00085 & $\begin{array}{l}\text { Amino sugar metabolism, fructose and mannose degradation, gluconeogenesis, glycolysis, pentose phosphate } \\
\text { pathway }\end{array}$ \\
\hline Glycerol 3-phosphate/Glycerol & Lipid metabolism & $5.41 E-04$ & 1.2 & 2.2 & $0.06(0.019,0.122)$ & $0.13(0.089,0.153)$ & HMDB00126/HMDB00131 & $\mathrm{C} 00093 / \mathrm{C} 00116$ & Glycerolipid metabolism \\
\hline D-Glucuronic acid & Carbohydrates & 7.63E-04 & 1.1 & 0.3 & $90,337.5(16,006.25,165,173.25)$ & $23,810.5(12,463,53,869.25)$ & HMDB00127 & Co0191 & Inositol Metabolism, starch and sucrose metabolism \\
\hline Glycerol & Alcohols & 8.09E-04 & 1.4 & 0.6 & $598,034.5(388,745.25,1,166,327)$ & $387,146(312,706.75,526,477)$ & HMDB00131 & C00116 & Galactose metabolism, glycerolipid metabolism \\
\hline Fumaric acid & Organic acids & $1.05 E-06$ & 2 & 0.6 & $8,265(6,316,10,364.25)$ & $4,577.5(3,857.5,5,716)$ & HMDB00134 & C00122 & $\begin{array}{l}\text { Arginine and proline metabolism, aspartate metabolism, itric acid cycle, mitochondrial electron transport } \\
\text { chain, phenylalanine and tyrosine metabolism, tyrosine metabolism, urea cycle }\end{array}$ \\
\hline Glyceric acid & Organic acids & $6.86 \mathrm{E}-04$ & 1.5 & 0.7 & $47,358(37,608.75,59,286.25)$ & $35,250(29,520.75,44,127.5)$ & HMDB00139 & C00258 & Glycerolipid Metabolism, Glycine and Serine Metabolism \\
\hline L-Glutamic acid & Amino acid & $2.28 E-06$ & 1.5 & 1.5 & $279,299.5(167,237.25,397,382)$ & $426,439.5(312,734.5,578,261)$ & HMDB00148 & C00025 & $\begin{array}{l}\text { Alanine metabolism, amino sugar metabolism, ammonia recycling, Arginine and proline metabolism, cysteine } \\
\text { metabolism, folate metabolism, glucose-alanine cycle, glutamate metabolism, glutathione metabolism, glycine } \\
\text { and serine metabolism, histidine metabolism, malate-aspartate shuttle, transcription/translation, urea cycle }\end{array}$ \\
\hline L-Glutamic acid/Oxoglutaric acid & Amino acid metabolism & $4.25 E-04$ & 1.4 & 2.7 & $25.75(20.325,42.947)$ & $69.01(40.097,153.817)$ & HMDB00148/HMDB00208 & C00025/C00026 & Arginine biosynthesis \\
\hline L-Glutamic acid/Pyroglutamic acid & Metabolism of other amino acids & $3.38 E-12$ & 2.1 & 2.2 & $0.14(0.092,0.174)$ & $0.3(0.222,0.384)$ & HMDB00148/HMDB00267 & $\mathrm{C} 00025 / \mathrm{C} 01879$ & Glutathione metabolism \\
\hline Hypoxanthine & Nucleotide & $3.00 E-03$ & 1.2 & 0.7 & $44,123.5(28,191,77,664.75)$ & $29,734.5(22,674.75,39,268.75)$ & HMDB00157 & C00262 & Purine Metabolism \\
\hline L-Tyrosine/L-Phenylalanine & Amino acid metabolism & $1.00 E-03$ & 1.5 & 0.6 & $1.87(1.318,2.323)$ & $1.21(0.861,1.49)$ & HMDB00158/HMDB00159 & C00082/C00079 & Phenylalanine metabolism; Phenylalanine, tyrosine and tryptophan biosynthesis \\
\hline L-Phenylalanine & Amino acid & 3.02E-06 & 1.6 & 1.4 & $433,113(323,081.25,575,814)$ & 620,982.5 (530,573.75, 707,172.25) & HMDB00159 & C00079 & Phenylalanine and tyrosine metabolism, transcription/translation \\
\hline L-Proline & Amino acid & $8.32 E-04$ & 1.2 & 0.5 & $961,090.5(582,251.5,1,158,145)$ & $516,571(459,014.75,757,644.75)$ & HMDB00162 & c00148 & Arginine and proline metabolism, transcription/translation \\
\hline D-Maltose & Carbohydrates & $4.20 \mathrm{E}-04$ & 1.1 & 0.5 & $29,752(11,850.25,74,833)$ & $16,010.5(10,526.75,24,970.5)$ & HMDB00163 & C00208 & Starch and sucrose metabolism \\
\hline L-Threonine & Amino acid & $5.00 E-03$ & 1.2 & 0.9 & $280,966(212,282.5,433,769.75)$ & $248,005.5(192,284.5,298,326)$ & HMDB00167 & Co0188 & Glycine and serine metabolism, threonine and 2-oxobutanoate degradation, transcription/translation \\
\hline D-Mannose & Carbohydrates & 3.36E-04 & 1.3 & 1.4 & $1,056,861(494,489.5,1,326,627.5)$ & $1,442,658.5(905,777.75,2,018,569.5)$ & HMDB00169 & c00159 & Fructose and mannose degradation, galactose metabolism \\
\hline L-Isoleucine & Amino acid & $4.00 E-03$ & 1.2 & 0.8 & $321,465(283,167.75,441,564.5)$ & $271,347.5(220,161.75,359,676)$ & HMDB00172 & C00407 & Transcription/translation, valine, leucine and isoleucine degradation \\
\hline Maleic acid & Organic acids & $3.00 E-03$ & 1.4 & 0.9 & $13,293.5(11,825,16,123)$ & $11,344(10,363.5,13,526)$ & HMDB00176 & C01384 & NA \\
\hline L-Lysine & Amino acid & $7.57 \mathrm{E}-04$ & 1.5 & 0.8 & $640,215(549,289.25,689,639.5)$ & $515,362.5(424,460.5,599,079.5)$ & HMDB00182 & c00047 & Biotin metabolism, carnitine synthesis, Iysine degradation, transcription/translation \\
\hline L-Lactic acid & Organic acids & $1.15 \mathrm{E}-04$ & 1.3 & 1.7 & $1,806,745(1,201,846,2,235,344.25)$ & $3,155,801(1,616,732,4,879,156.25)$ & HMDB00190 & Co0186 & Gluconeogenesis; pyruvate metabolism \\
\hline L-Cystine & Amino acid & $2.00 E-03$ & 1.3 & 0.7 & $175,895(120,707.75,238,916)$ & $117,730.5(87,831.5,169,729.25)$ & HMDB00192 & C00491 & $\begin{array}{l}\text { Cysteine and methionine metabolism; metabolic pathways; ABC transporters; ferroptosis; protein digestion } \\
\text { and absorption }\end{array}$ \\
\hline Myoinositol & Alcohols & $6.18 E-05$ & 1.4 & 0.4 & $438,968.5(215,182.25,1,198,811.75)$ & $170,756.5(114,897.5,289,559.5)$ & HMDB00211 & $\mathrm{C} 00137$ & $\begin{array}{l}\text { Galactose metabolism, inositol metabolism, inositol phosphate metabolism, phosphatidylinositol phosphate } \\
\text { metabolism }\end{array}$ \\
\hline O-Phosphoethanolamine & Lipids & $2.29 \mathrm{E}-05$ & 1.5 & 0.6 & $24,043(19,236,30,112)$ & $14,389.5(10,347.75,21,184)$ & HMDB00224 & C00346 & Sphingolipid metabolism \\
\hline Taurine & Organic acids & $2.35 E-09$ & 1.9 & 1.7 & $177,245.5(93,433.75,227,747.5)$ & $309,364.5(206,290.25,385,725.5)$ & HMDB00251 & C00245 & Bile acid biosynthesis, taurine and hypotaurine metabolism \\
\hline Pyroglutamic acid & Amino acid & $3.00 \mathrm{E}-03$ & 1.1 & 0.7 & $1,992,442.5(1,415,175,2,640,418.75)$ & $1,439,163(1,185,021.5,1,782,831.75)$ & HMDB00267 & C01879 & Glutathione metabolism \\
\hline Sarcosine & Amino acid & $2.30 \mathrm{E}-02$ & 1.3 & 0.9 & $9,147(8,219.75,10,872.75)$ & $8519.5(7,869.5,8,961.25)$ & HMDB00271 & C00213 & Glycine and serine metabolism \\
\hline Sarcosine/Dimethylglycine & Amino acid metabolism & $9.00 E-03$ & 1.4 & 0.7 & $0.03(0.018,0.036)$ & $0.02(0.017,0.026)$ & HMDB00271/HMDB00092 & C00213/C01026 & Glycine, serine and threonine metabolism \\
\hline Uric acid & Organic acids & $4.00 E-03$ & 1.3 & 0.6 & $292,716.5(207,345,341,138.5)$ & $188,751.5(146,691.25,270,719.75)$ & HMDB00289 & C00366 & Purine metabolism \\
\hline VanillyImandelic acid & Organic acids & $5.37 \mathrm{E}-04$ & 1.2 & 0.6 & $530(327.75,2,513.5)$ & $318(220.25,445.75)$ & HMDB00291 & C05584 & Tyrosine metabolism \\
\hline 5-Hydroxylysine & Amino acid & $3.11 \mathrm{E}-04$ & 1.6 & 0.5 & $2,092.5(1,624.5,4,172.25)$ & $1,141.5(727,1,643.75)$ & HMDB00450 & C16741 & Lysine degradation; metabolic pathways \\
\hline Creatinine & Amino acid & $6.36 \mathrm{E}-04$ & 1.2 & 0.8 & $338,468(224,928.75,1,273,947.25)$ & $254,810.5(179,753.5,344,497.5)$ & HMDB00562 & C00791 & Arginine and proline metabolism; Metabolic pathways \\
\hline Galactonic acid & Carbohydrates & $2.29 \mathrm{E}-05$ & 1.4 & 0.3 & $44,632(17,963,138,125.5)$ & $15,075(11,043,31,052)$ & HMDB00565 & $\mathrm{c} 00880$ & Galactose metabolism; metabolic pathways; microbial metabolism in diverse environments \\
\hline L-Cysteine & Amino acid & $5.15 E-04$ & 1.4 & 0.7 & $60,307.5(43,832,82,471.5)$ & $44,044(34,087.25,56,694.75)$ & HMDB00574 & NA & $\begin{array}{l}\text { Cysteine metabolism, glutathione metabolism, glycine and serine metabolism, methionine metabolism, } \\
\text { pantothenate and CoA biosynthesis, taurine and hypotaurine metabolism, transcription/translation }\end{array}$ \\
\hline L-Glutamine & Amino acid & $2.28 \mathrm{E}-06$ & 1.8 & 1.2 & $2,323,035(1,899,199,2,747,632.5)$ & $2,845,450(2,567,941.25,3,055,334.25)$ & 5)HMDB00641 & C00064 & $\begin{array}{l}\text { Amino sugar metabolism, ammonia recycling, glutamate metabolism, phenylacetate metabolism, purine } \\
\text { metabolism, pyrimidine metabolism, transcription/translation, urea cycle }\end{array}$ \\
\hline L-Glutamine/L-Glutamic acid & $\begin{array}{l}\text { Carbohydrate metabolism; Amino acid } \\
\text { metabolism }\end{array}$ & $1.00 \mathrm{E}-03$ & 1.1 & 0.7 & $9.28(6.079,14.034)$ & $6.66(4.532,8.929)$ & HMDB00641/HMDB00148 & $\mathrm{C} 00064 / \mathrm{C} 00025$ & Glyoxylate and dicarboxylate metabolism; alanine, aspartate and glutamate metabolism \\
\hline L-Arabinose/L-Arabitol & Carbohydrate metabolism & $1.56 \mathrm{E}-05$ & 1.5 & 2.1 & $0.22(0.143,0.436)$ & $0.46(0.393,0.6)$ & HMDB00646/HMDB01851 & C00259/C00532 & Pentose and glucuronate interconversions \\
\hline Linoleic acid & Fatty acids & 3.60E-02 & 1.2 & 0.8 & $162,340.5(109,351.5,223,310)$ & $131,850(91,854.25,183,544.5)$ & HMDB00673 & C01595 & Alpha Linolenic Acid and Linoleic Acid Metabolism \\
\hline Mannitol & Carbohydrates & $1.54 \mathrm{E}-04$ & 1.3 & 0.3 & $429,528.5(98,217.25,1,399,185.25)$ & $116,745(50,414.5,306,783.75)$ & HMDB00765 & C00392 & Fructose and mannose metabolism; ABC transporters; phosphotransferase system (PTS) \\
\hline N-Acetyl-L-aspartic acid & Amino acid & $3.00 \mathrm{E}-03$ & 1.1 & 1.1 & $27,759(10,844,179,723.5)$ & $29,926.5(15,873.25,46,000.5)$ & HMDB00812 & $\mathrm{C} 01042$ & Aspartate metabolism \\
\hline Normetanephrine & Hormone & $5.00 E-03$ & 1.4 & 0.8 & $3,813.5(2,690.25,5,572.75)$ & $2,953(2,047.5,3,518.5)$ & HMDB00819 & C05589 & Tyrosine metabolism \\
\hline Threonic acid & Carbohydrates & $1.00 \mathrm{E}-03$ & 1.2 & 0.9 & $51,359.5(34,386,201,621.75)$ & $45,318.5(30,874.25,56,184)$ & HMDB00943 & C01620 & NA \\
\hline L-Sorbose & Carbohydrates & $6.97 \mathrm{E}-06$ & 1.7 & 1.7 & $214,365.5(172,933,306,600.5)$ & $370,879(293,727,451,143.5)$ & HMDB01266 & c08356 & NA \\
\hline L-Arabitol & Carbohydrates & $3.28 \mathrm{E}-07$ & 1.7 & 0.5 & $110,028(65,100.75,240,871)$ & $55,786(43,840.25,67,209)$ & HMDB01851 & C00532 & Pentose and glucuronate interconversions; metabolic pathways \\
\hline Benzoic acid & Organic acids & 3.16E-09 & 2 & 0.6 & $24,087(19,048.5,29,166.5)$ & $14,903(13,031.25,17,855.5)$ & HMDB01870 & $\mathrm{c} 00180$ & NA \\
\hline Ribonolactone & Carbohydrates & $1.81 \mathrm{E}-05$ & 1.4 & 0.3 & $27,147.5(15,144,77,587.5)$ & $8,821.5(4,725.5,14,265.25)$ & HMDB01900 & C02674 & NA \\
\hline Tetracosanoic acid & Fatty acids & $1.00 \mathrm{E}-03$ & 1.2 & 0.9 & $6,202.5(5,134.75,8,127.75)$ & $5,272(4,122,6,202)$ & HMDB02003 & C08320 & Beta oxidation of very long chain fatty acids \\
\hline m-Cresol & Phenols & $2.00 \mathrm{E}-03$ & 1.2 & 0.5 & $30,252(80,40.5,101,712.75)$ & $13,858(5,703.75,26,689.25)$ & HMDB02048 & C01467 & Toluene degradation; microbial metabolism in diverse environments; degradation of aromatic compounds \\
\hline Petroselinic acid & Organic acids & $9.00 \mathrm{E}-03$ & 1.3 & 0.8 & $513,132(322,569.25,801,835.25)$ & $416,994(267,109.5,580,415.25)$ & HMDB02080 & C08363 & NA \\
\hline Erythrose & Carbohydrates & $9.57 \mathrm{E}-09$ & 1.8 & 0.5 & $535,478.5(354,430.5,923,590)$ & $249,182.5(52,987,324,922.75)$ & HMDB02649 & C01796 & NA \\
\hline Isomaltose & Carbohydrates & $5.60 \mathrm{E}-04$ & 1.1 & 0.5 & $8,272.5(2,733.75,19,707.75)$ & $4,314(2,844,6,996.25)$ & HMDB02923 & C00252 & NA \\
\hline Erythritol & Carbohydrates & 3.46E-05 & 1.4 & 0.5 & $66013(49,723,173,745.75)$ & $32,681(25,459.25,42,582.5)$ & HMDB02994 & C00503 & NA \\
\hline D-Threitol & Carbohydrates & $3.04 \mathrm{E}-05$ & 1.5 & 0.4 & $13,649.5(8,991,51,131.5)$ & $5,438.5(3,241.5,7,278.5)$ & HMDB04136 & C16884 & NA \\
\hline 5-Hydroxydopamine & Phenols & $4.20 E-02$ & 1.1 & 0.8 & $6,983.5(4,712.75,9,486)$ & $5617.5(3,889,7,842.5)$ & HMDB04817 & NA & NA \\
\hline MG160 & Lipids & $8.20 \mathrm{E}-06$ & 1.3 & 2.6 & $3,829(2,292.75,5,679.5)$ & $9,908(7,105.75,14,048)$ & HMDB11564 & NA & NA \\
\hline Glycerol 1-octadecanoate & Esters & $2.45 E-14$ & 2.3 & 2.9 & $3,563.5(2,668.5,4,804.75)$ & $10,494.5(8,536,12,954)$ & HMDB31075 & NA & NA \\
\hline
\end{tabular}

Differential metabolites were selected according to VIP $>1$ and P<0.05. Values are expressed as medians (IQRs). P values were calculated from t test for continuous variables and adjusted by FDR method; FC, fold change; VIP. variable influence on projection. 
Table S3 Differential urinary metabolites betweenT2DM with and without nephropathy.

\begin{tabular}{|c|c|c|c|c|c|c|c|c|c|}
\hline Name & Class & T test. P & VIP & FC & DN & T2DM & HMDBID & Kegg ID & Pathway \\
\hline D-Glucose & Carbohydrates & $2.12 \mathrm{E}-10$ & 3.0 & 0.3 & $267,788.5(181,641.25,363,846.25)$ & $89,577(41,878.25,157,795.5)$ & HMDB00122 & c00031 & $\begin{array}{l}\text { Galactose metabolism, gluconeogenesis, glucose-alanine cycle, } \\
\text { glycolysis, lactose degradation, Lactose synthesis, transfer of acetyl } \\
\text { groups into mitochondria }\end{array}$ \\
\hline L-Valine & Amino Acid & $9.40 \mathrm{E}-07$ & 2.9 & 0.3 & $14,824.5(9,025.25,31,585.5)$ & $4,716(3,303.5,7,020.5)$ & HMDB00883 & C00183 & $\begin{array}{l}\text { Propanoate metabolism, transcription/translation, valine, leucine and } \\
\text { isoleucine degradation }\end{array}$ \\
\hline L-Histidine & Amino Acid & 2.44E-05 & 2.4 & 0.3 & $10,364(4,639.75,18,147.25)$ & $3,146(899.25,4,270.5)$ & HMDB00177 & C00135 & Ammonia recycling, histidine metabolism, transcription/translation \\
\hline Sucrose & Carbohydrates & $6.89 \mathrm{E}-05$ & 2.3 & 0.2 & $1,200,070(410,986.25,1,750,933.5)$ & $267,627.5(108,851.5,665,185.75)$ & HMDB00258 & C00089 & Galactose metabolism, starch and sucrose metabolism \\
\hline Glycine & Amino Acid & 1.03E-04 & 2.2 & 0.6 & $65,575(39,493.75,105,077)$ & $39,768(20,924,53,651.75)$ & HMDB00123 & C00037 & $\begin{array}{l}\text { Alanine metabolism, ammonia recycling, bile acid biosynthesis, carnitine } \\
\text { synthesis, glutathione metabolism, glycine and serine metabolism, } \\
\text { methionine metabolism, porphyrin metabolism }\end{array}$ \\
\hline L-Asparagine/L-Aspartic acid & Amino acid metabolism & $1.71 \mathrm{E}-04$ & 2.0 & 0.5 & $9.68(5.958,20.118)$ & $4.66(2.934,7.522)$ & HMDB00168/HMDB00191 & C00152/C00049 & Alanine, aspartate and glutamate metabolism \\
\hline Gluconic acid & Carbohydrates & 3.60E-05 & 2.0 & 0.6 & $382,496.5(230,424.25,531,628.25)$ & $227,164.5(124,367.75,323,271.75)$ & HMDB00625 & C00257 & $\begin{array}{l}\text { Pentose phosphate pathway; metabolic pathways; biosynthesis of } \\
\text { secondary metabolites; microbial metabolism in diverse environments; } \\
\text { biosynthesis of antibiotics; carbon metabolism }\end{array}$ \\
\hline L-Cystine & Amino Acid & $1.00 \mathrm{E}-03$ & 2.0 & 0.3 & $133,144.5(72,901,283,660.5)$ & $44,629(16,423,104,929.5)$ & HMDB00192 & C00491 & $\begin{array}{l}\text { Cysteine and methionine metabolism; metabolic pathways; } \mathrm{ABC} \\
\text { transporters; ferroptosis; protein digestion and absorption }\end{array}$ \\
\hline L-Alanine & Amino Acid & $1.50 \mathrm{E}-02$ & 1.9 & 0.6 & $58,922.5(29,373.75,95,954.75)$ & $32,996(19,712.25,52,954)$ & HMDB00161 & C00041 & $\begin{array}{l}\text { Alanine metabolism, glucose-alanine cycle, glycine and serine } \\
\text { metabolism, selenoamino acid metabolism, transcription/translation, } \\
\text { urea cycle }\end{array}$ \\
\hline L-Asparagine & Amino Acid & 4.00E-03 & 1.9 & 0.5 & $148,956(71,175.5,246,210.25)$ & $67,998(39,736.5,132,908.75)$ & HMDB00168 & C00152 & Ammonia recycling, aspartate metabolism, transcription/translation \\
\hline L-Arabinose/L-Arabitol & Carbohydrate metabolism & $7.82 \mathrm{E}-04$ & 1.8 & 0.4 & $3.27(1.428,6.406)$ & $1.17(0.668,2.598)$ & HMDB00646/HMDB01851 & C00259/C00532 & Pentose and glucuronate interconversions \\
\hline 4-Hydroxybenzoic acid & Organic Acids & 4.00E-03 & 1.7 & 0.6 & 40,798.5 (21,313.75, 115,454.25) & $23,748.5(14,890.5,54,047)$ & HMDB00500 & C00156 & Ubiquinone biosynthesis \\
\hline p-Hydroxyphenylacetic acid & Organic Acids & 8.24E-04 & 1.7 & 1.3 & 194,089.5 (116,303, 313,946.25) & $261,616.5(167,321.75,786,099.25)$ & HMDB00020 & C00642 & Tyrosine metabolism \\
\hline L-Threonine & Amino Acid & $1.50 \mathrm{E}-02$ & 1.7 & 1.1 & $61,327(28,033.75,150,853)$ & $66,284(28,502,105,775)$ & HMDB00167 & C00188 & $\begin{array}{l}\text { Glycine and serine metabolism, threonine and 2-oxobutanoate } \\
\text { degradation, transcription/translation }\end{array}$ \\
\hline L-Phenylalanine/Phenylpyruvic acid & Amino acid metabolism & $6.13 \mathrm{E}-04$ & 1.7 & 0.3 & $30.1(11.584,61.208)$ & $8.09(4.657,15.047)$ & HMDB00159/HMDB00205 & C00079/C00166 & Phenylalanine, tyrosine and tryptophan biosynthesis \\
\hline L-Serine & Amino Acid & $1.00 \mathrm{E}-02$ & 1.7 & 0.9 & $92,666(71,705.5,163,200)$ & $79,442.5(36,488,107,665.25)$ & HMDB00187 & C00065 & $\begin{array}{l}\text { Ammonia recycling, glycine and serine metabolism, homocysteine } \\
\text { degradation, methionine metabolism, sphingolipid metabolism }\end{array}$ \\
\hline Galactonic acid & Carbohydrates & $1.00 \mathrm{E}-03$ & 1.6 & 0.7 & $54,048(37,692.25,93,398.25)$ & $35,796.5(19,514.75,57,736)$ & HMDB00565 & C00880 & $\begin{array}{l}\text { Galactose metabolism; metabolic pathways; microbial metabolism in } \\
\text { diverse environments }\end{array}$ \\
\hline Phenylpyruvic acid/L-Phenylalanine & Amino acid metabolism & $1.20 \mathrm{E}-02$ & 1.6 & 3.7 & $0.03(0.016,0.088)$ & $0.12(0.066,0.215)$ & HMDB00205/HMDB00159 & C00166/C00079 & Phenylalanine metabolism \\
\hline D-Glucose/Trehalose & Carbohydrate metabolism & $9.97 \mathrm{E}-04$ & 1.6 & 0.3 & $4.64(2.208,8.466)$ & $1.5(0.677,2.537)$ & HMDB00122/HMDB00975 & $\mathrm{C} 00031 / \mathrm{C} 01083$ & Starch and sucrose metabolism \\
\hline Xanthine & Nucleotide & $7.00 \mathrm{E}-03$ & 1.6 & 1.7 & $8,609.5(5,751.25,12,169.5)$ & $15,018(7,303.5,26,031.75)$ & HMDB00292 & C00385 & Purine metabolism \\
\hline D-Galactose & Carbohydrates & 2.00E-03 & 1.6 & 0.6 & $247,989.5(167,010.5,353,676.75)$ & $151,497(88,382,237,218.75)$ & HMDB00143 & Coo984 & $\begin{array}{l}\text { Galactose metabolism, lactose degradation, nucleotide sugars } \\
\text { metabolism }\end{array}$ \\
\hline Ornithine/L-Arginine & Amino acid metabolism & 2.20E-02 & 1.5 & 0.7 & $33.3(9.814,65.809)$ & $23.33(14.106,29.792)$ & HMDB00214/HMDB00517 & C00077/C00062 & Arginine biosynthesis; arginine and proline metabolism \\
\hline L-Lactic acid & Organic Acids & $4.50 \mathrm{E}-02$ & 1.5 & 0.5 & $430,096.5(201,968.75,737,092.5)$ & $200,346.5(91,188.75,407,080)$ & HMDB00190 & Co0186 & Gluconeogenesis, pyruvate metabolism \\
\hline Oxalic acid & Organic Acids & 4.63E-04 & 1.4 & 0.5 & $15,214.5(7,031.5,29,221.25)$ & $6,881.5(4,141.25,9,998)$ & HMDB02329 & C00209 & $\begin{array}{l}\text { Purine metabolism; chloroalkane and chloroalkene degradation; } \\
\text { glyoxylate and dicarboxylate metabolism; metabolic pathways; microbial } \\
\text { motabolism in diverse environments }\end{array}$ \\
\hline D-Ribose & Carbohydrates & $5.00 \mathrm{E}-03$ & 1.4 & 0.7 & $304,110.5(148,998.75,452,514.75)$ & $199,112(160,690.25,289,500.5)$ & HMDB00283 & Co0121 & Pentose phosphate pathway \\
\hline L-Glutamic acid & Amino Acid & 3.60E-02 & 1.4 & 1.0 & $29,932.5(18,307,60,635)$ & $29,173.5(15,021.5,48,765.75)$ & HMDB00148 & C00025 & $\begin{array}{l}\text { Alanine metabolism, amino sugar metabolism, ammonia recycling, } \\
\text { arginine and proline metabolism, cysteine metabolism, folate } \\
\text { metabolism, glucose-alanine cycle, glutamate metabolism, glutathione } \\
\text { metabolism, glycine and serine metabolism, histidine metabolism, } \\
\text { malate-aspartate shuttle, transcription/translation, urea cycle }\end{array}$ \\
\hline Hippuric acid & Organic Acids & $2.50 \mathrm{E}-02$ & 1.4 & 0.7 & $1,331,521(545,393.5,2,606,043)$ & $928,768(330,140.25,1,618,823.25)$ & HMDB00714 & C01586 & Phenylalanine metabolism \\
\hline Cytidine & Nucleotide & $2.00 \mathrm{E}-02$ & 1.4 & 0.4 & $434.5(148.5,720)$ & $174.5(84.75,439.25)$ & HMDB00089 & C00475 & Pyrimidine metabolism \\
\hline L-Xylonate & Organic Acids & 2.50E-02 & 1.4 & 0.6 & $319,783.5(226,142.25,471,741.5)$ & $192,075.5(124,227.5,342,688)$ & HMDB60256 & C05411 & $\begin{array}{l}\text { Pentose and glucuronate interconversions; ascorbate and aldarate } \\
\text { metabolism }\end{array}$ \\
\hline Mannitol & Carbohydrates & $3.00 \mathrm{E}-03$ & 1.4 & 1.6 & $257,485.5(177,979,411,349.25)$ & 399,367 (266,932.25, 641,879.75) & HMDB00765 & C00392 & $\begin{array}{l}\text { Fructose and mannose metabolism; ABC transporters; } \\
\text { phosphotransferase system (PTS) }\end{array}$ \\
\hline Normetanephrine & Hormone & $1.50 \mathrm{E}-02$ & 1.4 & 1.6 & $5,526(2,798.5,10,694.25)$ & $8,911.5(3,673.25,19,318.5)$ & HMDB00819 & C05589 & Tyrosine Metabolism \\
\hline Uridine & Nucleotide & $1.50 \mathrm{E}-02$ & 1.4 & 0.6 & $3,777(2,104.75,6,605.5)$ & $2,230.5(1,601.25,4,315.25)$ & HMDB00296 & C00299 & Pyrimidine Metabolism \\
\hline D-Fructose/Sucrose & Carbohydrate metabolism & $1.20 \mathrm{E}-02$ & 1.3 & 2.1 & $0.11(0.059,0.259)$ & $0.23(0.071,0.763)$ & HMDB00660/HMDB00258 & C02336/C00089 & Starch and sucrose metabolism \\
\hline D-Threitol & Carbohydrates & $8.00 \mathrm{E}-03$ & 1.3 & 0.8 & $45,409.5(31,440,66,992.5)$ & $34,121(19,136,47,399.5)$ & HMDB04136 & C16884 & NA \\
\hline Ketoleucine & Amino Acid & $8.00 \mathrm{E}-03$ & 1.3 & 2.8 & $8,137(4,966,16,225.25)$ & $22,470.5(4,557.75,49,945.5)$ & HMDB00695 & $\mathrm{C} 00233$ & Valine, leucine and isoleucine degradation \\
\hline 1,2,3-Trihydroxybenzene & Phenols & 3.50E-02 & 1.3 & 1.4 & 23,996 (6,720, 48,837) & $33,284.5(8,821.25,85,409.75)$ & HMDB13674 & C01108 & $\begin{array}{l}\text { Aminobenzoate degradation; microbial metabolism in diverse } \\
\text { environments; catecholamine transferase inhibitors }\end{array}$ \\
\hline Uracil/Uridine & Nucleotide metabolism & $1.70 \mathrm{E}-02$ & 1.2 & 2.2 & $2.01(0.726,3.555)$ & $4.38(1.92,9.464)$ & HMDB00300/HMDB00296 & C00106/C00299 & Pyrimidine metabolism \\
\hline Hydroxyphenyllactic acid & Organic Acids & 2.40E-02 & 1.2 & 2.1 & $83,610(39,609.25,132,933.5)$ & $178,418.5(75,759,275,474.25)$ & HMDB00755 & C03672 & NA \\
\hline Citramalic acid & Organic Acids & 3.00E-02 & 1.2 & 0.5 & 20,538.5 (9,175.25, 43,236.75) & $11,130.5(6,810.25,25,437.25)$ & HMDB00426 & C00815 & NA \\
\hline L-Arabinose & Carbohydrates & $2.90 \mathrm{E}-02$ & 1.2 & 0.8 & 442,924 (337,962, 666,296.25) & $350,838.5(184,538.25,468,567.25)$ & HMDB00646 & C00259 & $\begin{array}{l}\text { Pentose and glucuronate interconversions; ascorbate and aldarate } \\
\text { metabolism; amino sugar and nucleotide sugar metabolism; metabolic } \\
\text { pathways; ABC transporters }\end{array}$ \\
\hline D-Fructose & Carbohydrates & $1.70 \mathrm{E}-02$ & 1.2 & 0.6 & $104,449.5(49,230.25,185,290)$ & $64,712.5(30,731.75,106,221)$ & HMDB00660 & C02336 & $\begin{array}{l}\text { Amino sugar metabolism, fructose and mannose degradation, galactose } \\
\text { metabolism, starch and sucrose metabolism }\end{array}$ \\
\hline Inosine/Adenosine & Nucleotide metabolism & $1.30 \mathrm{E}-02$ & 1.1 & 1.5 & $0.35(0.19,0.599)$ & $0.51(0.301,1.445)$ & HMDB00195/HMDB00050 & C00294/C00212 & Purine metabolism \\
\hline Inosine & Nucleotide & $2.50 \mathrm{E}-02$ & 1.1 & 1.6 & $2,835.5(1,850,5,864.75)$ & 4,552.5 (2,281, 14,824.75) & HMDB00195 & C00294 & Purine metabolism \\
\hline
\end{tabular}



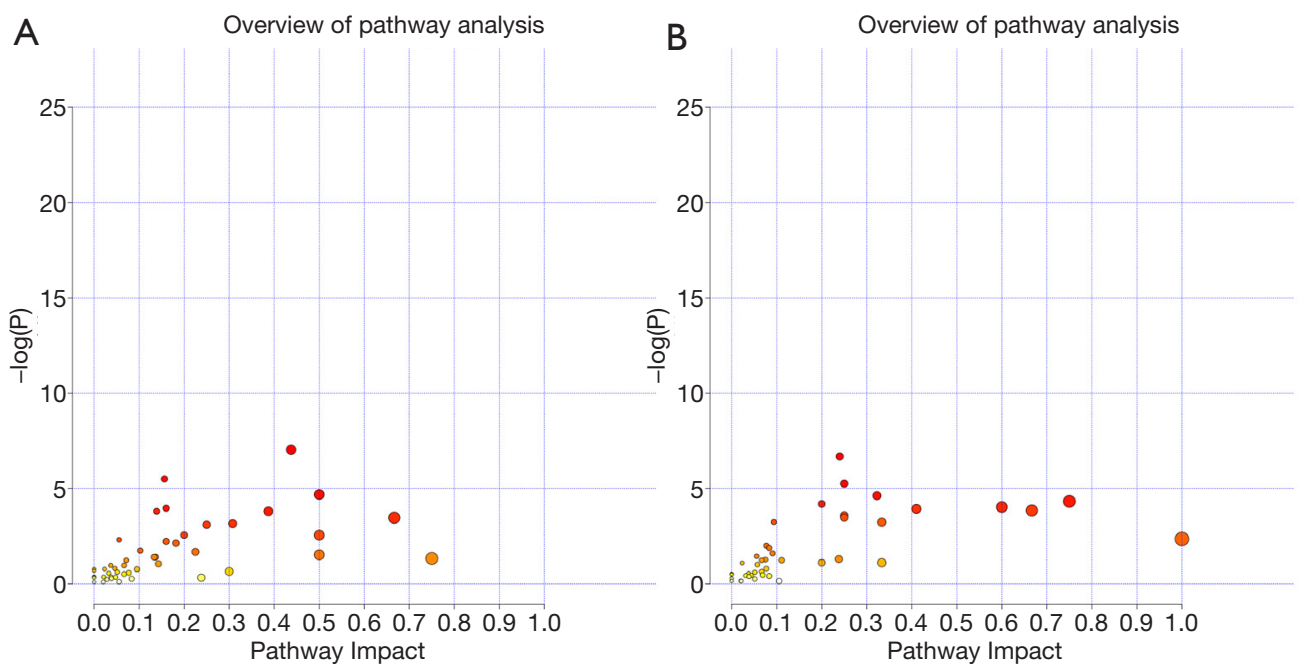

Figure S2 Metabolic pathway analysis. (A) Serum metabolic pathway analysis. (B) Urine metabolic pathway analysis. All the matched pathways are displayed as circles. The colour and size of each circle are based on the $\mathrm{P}$ value and pathway impact value, respectively. The graph was obtained by plotting on the $\mathrm{y}$-axis the $\log$ of $\mathrm{P}$ values from the pathway enrichment analysis and on the $\mathrm{x}$-axis the pathway impact values derived from the pathway topology analysis. 